\title{
Signed enumeration of ribbon tableaux: an approach through growth diagrams
}

\author{
Dominique Gouyou-Beauchamps • \\ Philippe Nadeau
}

Received: 20 May 2010 / Accepted: 10 October 2011 / Published online: 10 November 2011

(C) Springer Science+Business Media, LLC 2011

\begin{abstract}
We give an extension of the famous Schensted correspondence to the case of ribbon tableaux, where ribbons are allowed to be of different sizes. This is done by extending Fomin's growth diagram approach of the classical correspondence, in particular by allowing signs in the enumeration. As an application, we give in particular a combinatorial proof, based on the Murnaghan-Nakayama rule, for the evaluation of the column sums of the character table of the symmetric group.
\end{abstract}

Keywords Ribbon tableaux · Growth diagrams · Murnaghan-Nakayama rule · Garsia-Milne involution principle $\cdot$ RSK correspondence

\section{Introduction}

The Schensted correspondence [22] is a bijection between permutations and pairs of standard Young tableaux of the same shape. It has been extended in numerous ways, the most famous being certainly the Robinson-Schensted-Knuth correspondence between matrices of integers and pairs of semi-standard tableaux of the same shape. Other extensions exist, for instance, oscillating tableaux [2-4, 29], skew tableaux [21], shifted Young tableaux [19], and $k$-ribbon tableaux [23, 28].

Sergey Fomin developed a general theory of such correspondences, cf. [5-9]. It unifies the correspondences listed above by interpreting these tableaux as paths in so-called graded graphs. For instance, a Young tableau in this context is viewed as a particular kind of a path in the graph whose vertices are integer partitions, and

D. Gouyou-Beauchamps

Laboratoire de Recherche en Informatique, Université Paris Sud, 91405 Orsay, France e-mail: dgb@acces.lri.fr

P. Nadeau ( $\square)$

Fakultät für Mathematik, Universität Wien, Garnisongasse 3, 1090 Vienna, Austria

e-mail: philippe.nadeau@univie.ac.at 
where $(\lambda, \mu)$ is an edge if $\mu$ is a partition obtained after adding 1 to a part of $\lambda$. This graph is usually called the Young graph (or Young lattice). Other combinatorial objects can then be represented by considering other paths, for instance, by modifying the extreme points of the path, or the edges that one can use. This is a way of interpreting oscillating or skew tableaux inside the Young graph, for instance. Then the local properties of the graph will give rise to various bijective correspondences, all consequences of one elementary bijection.

Furthermore, Fomin gives in parallel a linear algebraic approach to his results, which is directly inspired by the work of Stanley [25]. As a matter of fact, most of Fomin's results have both a bijective and an algebraic proof.

In [31], Dennis White describes another extension of the Schensted correspondence for ribbon tableaux where ribbons are allowed to have different sizes; his goal was, in fact, to give a combinatorial proof of the second orthogonality relation for characters of the symmetric group. The algorithm describing his correspondence is a complicated insertion mechanism, along the lines of the original Schensted correspondence; this forces him moreover to put some restrictions on the ribbon tableaux he considers.

In this work, we will show how both the bijective and algebraic approaches of Fomin $[6,7]$ can be adapted to apply to the correspondence of White; the main results are gathered in Sect. 7. There will be two advantages to this: First, this will clarify the original insertion algorithm of White and extend it at the same time. Then, we will in the process have to extend Fomin's setting of dual graded graphs to graphs which are more general. Although we will be mainly considering the case of ribbon tableaux, we will define in the last section the type of graphs to which our work can be generalized. Note also that we will deal here with signed objects, i.e., with weights plus or minus one: it will appear that, for the bijective approach, we make use the famous involution principle of Garsia and Milne [12].

The correspondence we establish here is between pairs of ribbon tableaux of the same shape, on the one side, and so-called hook permutations. It should be noted that the most famous property of the usual Schensted correspondence, namely that exchanging the two standard tableaux results in inverting the permutation, does not hold in our extension. A special case of this property subsists however, that is, the fact that standard tableaux are in bijection with involutions can be generalized to ribbon tableaux and hook involutions, see Theorem 7.2.

The paper is organized as follows: In Sect. 2, we give the main definitions about ribbons and ribbon tableaux, as well as some elementary operations on these objects. In Sect. 3, we define different notions about signed enumeration, and we recall the involution principle of Garsia and Milne. Section 4 introduces hook permutations which are the objects in correspondence with pairs of ribbon tableaux in our main result.

The description of the bijections is given in the two following sections: Sect. 5 describes local rules based on the operations from Sect. 2, and Sect. 6 shows how to define a global correspondence from these local rules. This is directly inspired by [7]. Section 7 states the main results, which are Theorems 7.1 and its corollary, and Theorem 7.2: there exists a signed bijection between hook permutations and pairs of ribbon tableaux of the same shape, and a signed bijection between hook involutions 
and ribbon tableaux. The proofs of Theorems 7.1 and 7.2 are given in Sect. 8. In Sect. 9, a linear algebraic version of Theorem 7.1 is given.

Section 10 contains an application of Theorem 7.2 to the column sums of the character table of the symmetric group. Finally, Sect. 11 explains how the methods developed can be used for other enumerations, and details in what ways this bijective and algebraic setting is a generalization of Sergey's Fomin graded graphs in duality.

\section{Ribbons}

\subsection{Definitions}

A partition $\lambda=\left(\lambda_{1}, \ldots, \lambda_{m}\right)$ is a nonincreasing finite sequence of positive integers; these integers are the parts of the partition, the size of the partitions being their sum $|\lambda|:=\sum_{i} \lambda_{i}$. A composition $c$ is a finite sequence of positive integers; we can associate to $c$ a partition $\widetilde{c}$ by rearranging the integers in nonincreasing order. A partition $\lambda$ can be described using an exponential notation $\lambda=\left[1^{j_{1}}, 2^{j_{2}}, \ldots\right]$, where $j_{i}$ is the number of parts of size $i$. If $j_{i}=0$, then $i^{j_{i}}$ is not written and if $j_{i}=1, j_{i}$ is not written.

We will identify a partition $\left(\lambda_{1}, \ldots, \lambda_{m}\right)$ with its Ferrers diagram, which is the left justified set of cells (i.e., unit squares of $\mathbb{Z}^{2}$ ) such that the $i$ th row from the top contains $\lambda_{i}$ cells; the diagram on the left of Fig. 1 represents the partition $(8,6,5,2,2,1,1)=\left[1^{2} 2^{2} 568\right]$.

Let $\mathbf{Y}$ be the set of integer partitions, and $\mathbf{Y}_{n}$ the subset of partitions of size $n$. Two partitions $\lambda \subseteq \mu$ (in the sense of inclusion of Ferrers diagrams) define a skew shape $\mu / \lambda$. We will identify here a skew shape with the set of cells $\mu \backslash \lambda$, whenever $\mu$ or $\lambda$ is clear from the context; though, in general, two distinct skew shapes may define the same set, this will not create any ambiguity here. The size of $\mu / \lambda$ is its number of cells $|\mu|-|\lambda|$ and will be noted $|\mu / \lambda|$. The skew shape $(9,8,7,4,4,1,1) /(8,6,5,2,2,1,1)$ represented on the right of Fig. 1 has size 9.

Let us say that a subset $S$ of cells of $\mathbb{Z}^{2}$ is connected if, for every two cells $c, c^{\prime}$ in $S$, there exists a sequence of cells $c=c_{0}, c_{1}, \ldots, c_{t}=c^{\prime}$ in $S$ such that $c_{i}, c_{i+1}$ have a common side for all $i$. We can then define the notion of ribbons:

Definition 2.1 A ribbon is a nonempty connected skew shape that does not contain a 2 by 2 square of cells.
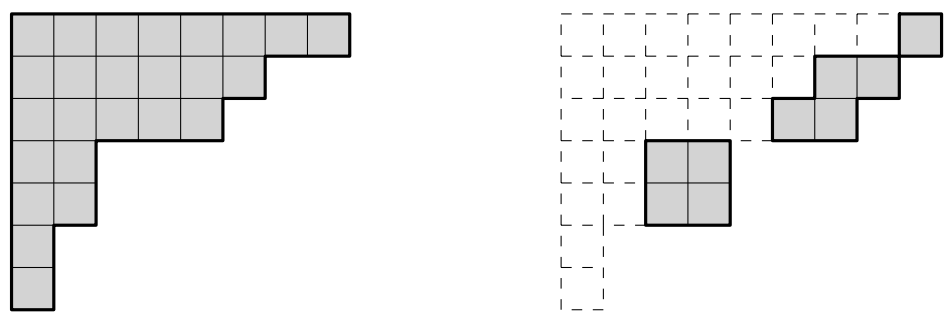

Fig. 1 Partition and skew shape 

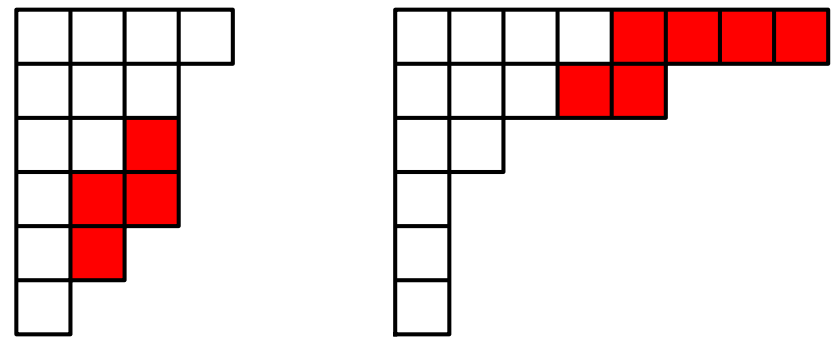

Fig. 2 Examples of ribbons

Fig. 3 First levels of the ribbon graph $G R$

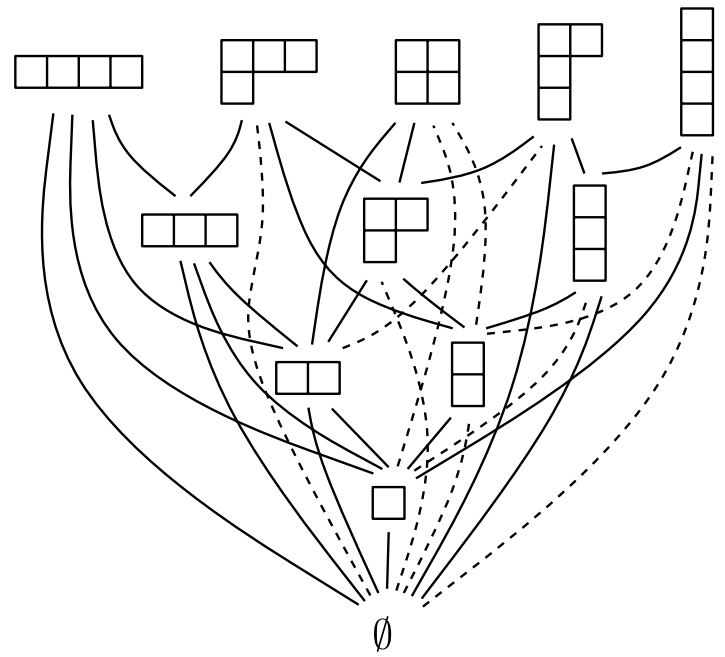

Let $r=\mu / \lambda$ be a ribbon. Such a ribbon is said to be $\mu$-addable and $\lambda$-removable. Its height $h(r)$ is defined as the number of lines it occupies minus one, and its sign is then $\varepsilon(r):=(-1)^{h(r)}$. The bottom left cell of $r$ is its tail, and the top right one is its head. Given a partition $\lambda$, the ribbons that can be removed or added to $\lambda$ are entirely determined by the coordinates of their heads and tails. There are two ribbons in Fig. 2: the left one has size 4, height 2 and sign +1 , and the right one has size 6 , height 1 and thus sign -1 .

A hook is a ribbon of shape $\lambda / \emptyset$, which is equivalently a partition of the kind $(k, 1, \ldots, 1)$ where $k \geq 1$. Note that a hook is characterized by the data of its size $s$ and height $h \in \llbracket 0, s-1 \rrbracket$.

For $i$ a positive integer, we denote by $R i b_{i}$ the set of ribbons of size $i$, and $R i b:=\bigcup_{i>0} R i b_{i}$. The set $\mathbf{Y}$ is made into an infinite graph $G R$, with an edge for each element of $R i b$; each edge carries in addition a sign which is simply the sign of the corresponding ribbon. We associate to each vertex of $G R$ a certain level given by the size of the corresponding partition. Figure 3 shows the first five such levels of $G R$, where the dotted edges represent negative ribbons. The addition (resp., removal) of a ribbon is then equivalent to a step up (resp., down) in $G R$. 


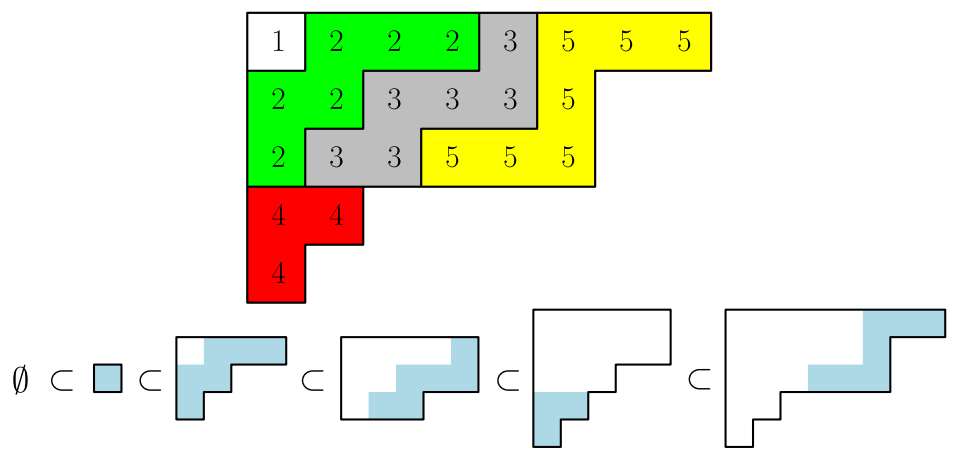

Fig. 4 Two equivalent representations of a ribbon tableau

Definition 2.2 A ribbon tableau of shape $\lambda \in \mathbf{Y}$ and length $\ell$ is a sequence of partitions $\lambda^{0}=\emptyset \subset \lambda^{1} \subset \cdots \subset \lambda^{\ell-1} \subset \lambda^{\ell}=\lambda$ such that $r_{i}:=\lambda^{i+1} / \lambda^{i}$ is a ribbon for every $i<\ell$.

We will often represent a ribbon tableau by a labeling of the cells of $\lambda$, in which the cells labeled $i$ coincide with the ribbon $r_{i}$. Note that a ribbon tableau is equivalently a path of length $\ell$ in the graph $G R$, going monotonically up from $\emptyset$ to $\lambda$; this interpretation is the key to the extensions described in Sect. 11.

We need some more definitions. The sign $\varepsilon(P)$ of a tableau $P$ is the product of the signs of the ribbons $r^{(i)}$. The content $c(P)$ is the composition of $|\lambda|$ in $\ell$ parts formed by the sequence of sizes $\left|r^{(1)}\right|, \ldots,\left|r^{(\ell)}\right|$. We will denote by $R T_{\lambda, c}$ the tableaux of shape $\lambda$ and content $c$, where $c$ is a given composition of $|\lambda|$ and $R T_{\lambda}(\ell)$ the set of all ribbon tableaux of shape $\lambda$ and length $\ell$.

Figure 4 shows a tableau of shape $(8,6,6,2,1)$, content $(1,6,6,3,7)$ and sign $(-1)^{0}(-1)^{2}(-1)^{2}(-1)^{1}(-1)^{2}=-1$.

\subsection{Operations on ribbons}

We will now introduce some classical operations on ribbons which are necessary for the definition of the local rules of Sect. 5.

- bumpin, bumpout: Let $\lambda$ be a partition, and $r, r^{\prime}$ be two ribbons that are $\lambda$-addable, such that $r$ and $r^{\prime}$ have different heads and different tails. Then

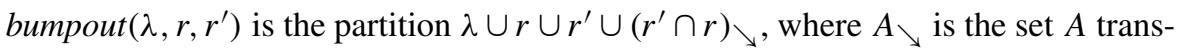
lated by one unit east and south. The partition $\operatorname{bumpin}\left(\xi, r, r^{\prime}\right)$ is similarly defined for two $\xi$-removable ribbons, by translating the common cells between $r$ and $r^{\prime}$ by the vector $(-1,1)$. Figure 5 shows the way this operation acts, according to the relative positions of $r$ and $r^{\prime}$ : they can be disjoint, or partially overlap, or one can be included in the other.

- prev, next, first: Let $\lambda$ be a partition, $k$ a positive integer, and $h$ a nonnegative integer. Let $\left(r_{i}\right)_{i=0, \ldots, t}$ (resp., $\left.\left(r_{i}^{\prime}\right)_{i=1, \ldots, t^{\prime}}\right)$ be the list of ribbons of size $k$ and height $h$ that are $\lambda$-addable (resp., $\lambda$-removable). Then we have the following result from Shimozono and White [23, Lemma 14]: 

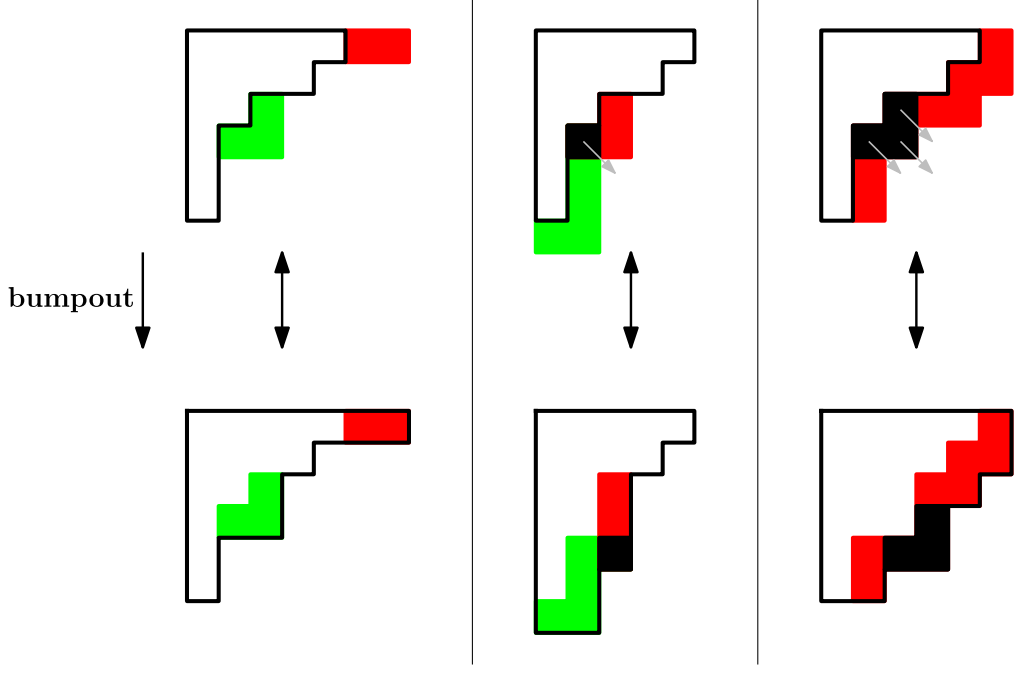

bumpin

Fig. 5 Operations bumpin and bumpout

Fig. 6 Addable and removable

ribbons of height 1 and size 3

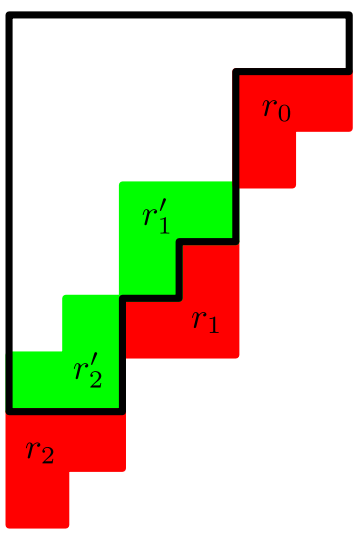

Proposition 2.1 With $\lambda, h, k,\left(r_{i}\right)_{i=0, \ldots, t},\left(r_{i}^{\prime}\right)_{i=1, \ldots, t^{\prime}}$ as above, we have then (i) $t=t^{\prime}$ and (ii) the enumeration order of the ribbons can be chosen so that $r_{0}<r_{1}^{\prime}<r_{1}<$ $\cdots<r_{t}^{\prime}<r_{t}$ where rib ${ }_{1}<r i b_{2}$ means that the head of rib $b_{1}$ is north east of the head of $r i b_{2}$.

Figure 6 illustrates Proposition 2.1. This allows defining certain operations first, next, and prev on ribbons:

- If $E q$ is a hook of size $k$ and height $h$, we define $f i r s t(\lambda, E q)$ as the ribbon $r_{0}$ above.

- If $r^{\prime}$ is a $\lambda$-removable ribbon, i.e., $r^{\prime}=r_{i}^{\prime}$ for a certain $i \in \llbracket 1, t \rrbracket$, then $\operatorname{next}\left(\lambda, r^{\prime}\right):=$ $\lambda \cup r_{i}$. 


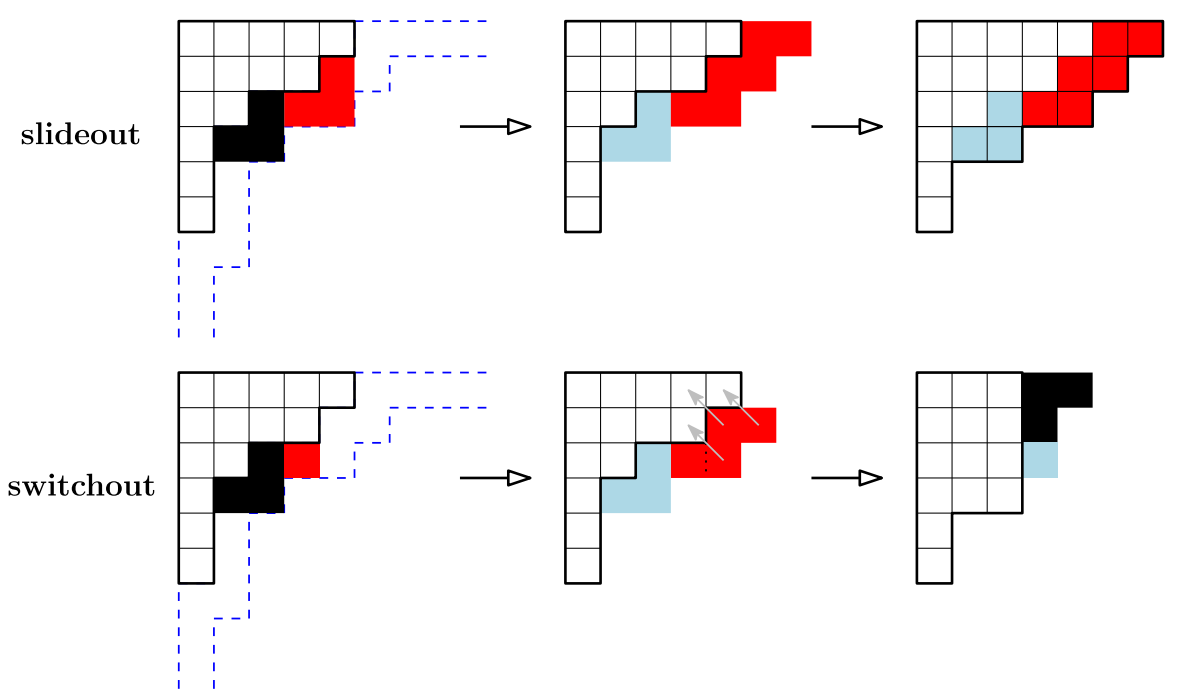

Fig. 7 Operations switchout and slideout

- Reciprocally, if $r=r_{i}$ for any $i \in \llbracket 1, t \rrbracket$ is a $\lambda$-addable ribbon, we define $\operatorname{prev}\left(\lambda, r_{i}\right):=\lambda r_{i}^{\prime}$, while $\operatorname{prev}\left(\lambda, r_{0}\right)$ is not defined.

- slideout, switchout, slidein, switchin: Let $\lambda$ be a partition, and $r, r^{\prime}$ two $\lambda$-addable ribbons with identical tails but different heads; we assume, without loss of generality, that $|r|>\left|r^{\prime}\right|$. The external band of $\lambda$ consists of all cells between the infinite south east boundary of $\lambda$ and its translation by $(1,-1)$, i.e., the cells enclosed by the dotted line on Fig. 7. Let $\tau$ be the subset of the external band formed by the $\left|r^{\prime}\right|$ connected cells, north west of $r$ and adjacent to it. Then two cases can occur:

- If $\tau \cup r$ forms a $\lambda$-addable ribbon, we define the partition $\operatorname{sideout}\left(\lambda, r, r^{\prime}\right)=\lambda \cup$ $r \cup \tau$.

- Otherwise, we define switchout $\left(\lambda, r, r^{\prime}\right)=\left(\lambda \cup r^{\prime}\right) \backslash \tau_{\nwarrow}$, where $\tau_{\nwarrow}$ is the translated of $\tau$ by the vector $(-1,1)$.

If $r$ and $r^{\prime}$ have the same head but different tails, one performs the same operations on the transposed partitions. The operations switchin and slidein are defined similarly on $\lambda$-removable ribbons; see White [31] for supplementary explanations.

\section{Signed sets and signed bijections}

In this work, we have to deal with signed enumerations, so we need some definitions and notations to explain what we mean by a bijection in this context. All sets are assumed to be finite.

Definition 3.1 (Signed sets) A signed set is a set $A$ together with a decomposition $A=A^{+} \cup A^{-}$where $A^{+} \cap A^{-}=\emptyset$. The members of $A^{+}$are positive elements, those of $A^{-}$are negative. 
Such a decomposition is equivalent to a function $\delta: A \rightarrow\{1,-1\}$, with the obvious correspondence $A^{+}=\delta^{-1}(\{1\})$ and $A^{-}=\delta^{-1}(\{-1\})$. Our objects of study here are the sets $R T_{\lambda, \mu}$, the sign being given by the function $\varepsilon$. Note also that usual sets are considered as positive sets. The signed cardinal (or signed sum) of a signed set $A$ is $|A|_{ \pm}=\left|A^{+}\right|-\left|A^{-}\right|$; if the sign is given by a function $\delta$, then we have $|A|_{ \pm}=$ $\sum_{a \in A} \delta(a)$.

A function $f$ between two signed sets is sign preserving (resp., sign reversing) if $a$ and $f(a)$ have the same sign (resp., opposite signs) for all $a$. Fixed points of a function $i$ form the set Fix $(i)$.

Definition 3.2 (Signed bijections) A signed bijection between two signed sets $A$ and $B$ is a triplet of functions $\left(i_{A}, i_{B}, \phi\right)$ such that $i_{A}$ (resp., $\left.i_{B}\right)$ is an involution on $A$ (resp., on $B$ ) which is sign reversing outside of its fixed point set, and $\phi$ is a sign preserving bijection between $\operatorname{Fix}\left(i_{A}\right)$ and $\operatorname{Fix}\left(i_{B}\right)$.

A signed bijection between $A$ and $B$ proves that $|A|_{ \pm}=|B|_{ \pm}$; indeed, we have

$$
|A|_{ \pm}=\left|\operatorname{Fix}\left(i_{A}\right)\right|_{ \pm}=\left|\operatorname{Fix}\left(i_{B}\right)\right|_{ \pm}=|B|_{ \pm} .
$$

The central equality comes from the sign preserving bijection $\phi$, the other ones from the fact that $i_{A}$ and $i_{B}$ are sign reversing, so the pairs $\left\{a, i_{A}(a)\right\}$ such that $a \neq$ $i_{A}(a)$ have a zero contribution to the signed cardinal of $A$, the same being true for $B$ and $i_{B}$.

Remark 3.1 $|A|_{ \pm}=|B|_{ \pm}$is obviously equivalent to $\left|A^{+}\right|+\left|B^{-}\right|=\left|B^{+}\right|+\left|A^{-}\right|$. Suppose $\psi$ is a bijection between the ordinary sets $A^{+} \sqcup B^{-}$and $B^{+} \sqcup A^{-}$; we will show why such a $\psi$ is equivalent to a signed bijection between $A$ and $B$.

Let $M_{A}$ be the set of elements $a \in A^{+}$such that $\psi(a) \in A^{-}$, and $M_{B}$ be the set of elements $b \in B^{-}$such that $\psi(b) \in B^{+}$. Then $\psi, \psi^{-1}$ induce sign reversing involutions on $M_{A} \cup \psi\left(M_{A}\right)$ and $M_{B} \cup \psi\left(M_{B}\right)$, as well as a sign-preserving correspondence between $A^{+}-M_{A}$ (resp., $B^{-}-M_{B}$ ) and $B^{+}-\psi\left(M_{B}\right)$ (resp., $A^{-}-\psi\left(M_{A}\right)$ ). Putting things together, we obtain a signed bijection between $A$ and $B$; the whole process is clearly reversible.

The involution principle of Garsia and Milne Garsia and Milne gave the first fully bijective proof of a combinatorial version of a famous Rogers-Ramanujan identity $[11,12]$ which states that the number of partitions $\left(\lambda_{1}, \ldots, \lambda_{k}\right)$ of $n$ verifying $\lambda_{i}-\lambda_{i+1} \geq 2$ for all $i<k$ is the same as those verifying $\lambda_{i} \equiv 1$ or 4 modulo 5 for all $i \leq k$. To achieve this, they defined and used a general principle that we now recall.

Let $A, B$ be two finite signed sets. Let also $i_{A}, i_{B}$ be two involutions on $A$ and $B$, respectively, and $\phi$ a bijection between $A$ and $B$. We suppose that $\phi$ preserves signs, whereas $i_{A}$ and $i_{B}$ reverse signs outside their fixed point sets.

Under these assumptions, one clearly has $\left|F i x\left(i_{A}\right)\right|_{ \pm}=\left|F i x\left(i_{B}\right)\right|_{ \pm}$, but one does not have an obvious signed bijection proving this equality. The principle of Garsia and Milne is the construction of such a signed bijection $\left(\psi, j_{A}, j_{B}\right)$ between $\operatorname{Fix}\left(i_{A}\right)$ and $F i x\left(i_{B}\right)$ in the following manner. Let $a \in \operatorname{Fix}\left(i_{A}\right)$. By applying to $a$ the functions $\phi: A \rightarrow B$, and then, alternatively, the functions $\phi^{-1} \circ i_{B}: B \rightarrow A$ and $\phi \circ i_{A}: A \rightarrow$ 
$B$, we obtain a sequence $x_{0}=a, x_{1}, x_{2}, \ldots$ of elements where $x_{2 p} \in A$ and $x_{2 p-1} \in B$ for $p>0$. Then let $q>0$ be minimal such that either $q$ is odd and $x_{q} \in$ Fix $\left(i_{B}\right)$, or $q$ is even and $x_{q} \in \operatorname{Fix}\left(i_{A}\right)$. In the first case, we set $\psi(a):=x_{q}$ and $j_{A}(a):=a$, otherwise we set $j_{A}(a):=x_{q}$.

To define $j_{B}$ (and $\psi^{-1}$ ), one uses the symmetric procedure starting from $b \in B$. These procedures terminate and give the desired signed bijection.

The name "involution principle of Garsia and Milne" is usually reserved to the special case where both $\operatorname{Fix}\left(i_{A}\right)$ and $\operatorname{Fix}\left(i_{B}\right)$ consist of positive elements, so that one constructs in the end an ordinary bijection. The proof of this special case can be found, for instance, in [14, p. 76]; the general case follows the same lines.

\section{Hook permutations}

We now introduce the notions of hook permutations: these play the role of the ordinary permutations in the Schensted correspondence in the main correspondence of Theorem 7.1.

If $H=\left(H_{1}, \ldots, H_{\ell}\right)$ is a sequence of $\ell$ hooks, its content $c(H)$ is the composition $\left(\left|H_{1}\right|, \ldots,\left|H_{\ell}\right|\right)$. The length of $H$ is $\ell$, and its size is $\sum_{i}\left|H_{i}\right|$.

Definition 4.1 A hook permutation $(H, \sigma)$ is a sequence $H=\left(H_{1}, \ldots, H_{\ell}\right)$ of $\ell$ hooks together with a permutation $\sigma$ of $\llbracket \ell \rrbracket$. We also define $\sigma(H)$ as $\left(H_{\sigma^{-1}(1)}, \ldots, H_{\sigma^{-1}(\ell)}\right)$.

We will write $\mathcal{H P}$ for the set of hook permutations, its elements of content $\mu$ forming $\mathcal{H} \mathcal{P}(\mu)$ (where $\mu$ is any composition). Hook permutations can be graphically represented by the list $H$ where the cells of hook $H_{i}$ are labeled by $\sigma(i)$, or by square arrays of size $\ell$ such that entry $(i, j)$ is empty unless $j=\sigma(i)$ in which case it is occupied by the $i$ th hook $H_{i}$. Illustrations are given in Fig. 8 .

We then have the following proposition:

Proposition 4.1 The number of hook sequences of length $\ell$ and total size $n$ is equal to $\left(\begin{array}{c}n+\ell-1 \\ 2 \ell-1\end{array}\right)$. The number of hook permutations of length $\ell$ and total size $n$ is equal to $\ell ! \cdot\left(\begin{array}{c}n+\ell-1 \\ 2 \ell-1\end{array}\right)$.

Proof The first assertion obviously implies the second one. Given such a hook sequence, we can associate to it $2 \ell-1$ integers in $\llbracket n+\ell-1 \rrbracket$, as illustrated graphically in Fig. 9, in which $n=23, \ell=5$, so $n+\ell-1=27$ and $2 \ell-1=9$, and the subset of integers is $\{4,7,9,12,13,19,21,22,26\}$.

Conversely, if we have $2 \ell-1$ integers $1 \leq i_{1}<\cdots<i_{2 \ell-1} \leq n+\ell-1$, and we set by convention $i_{0}=0$ and $i_{2 \ell}=n+\ell$, then the list of hooks $\left(H_{1}, \ldots, H_{\ell}\right)$ in bijection is characterized by the fact that $H_{i}$ is the hook of size $i_{2 i}-i_{2 i-2}-1$ and height $i_{2 i-1}-i_{2 i-2}-1$. This is clearly bijective, and the proof is complete.

In Sect. 6.2, we introduce "hook involutions", which contrary to hook permutations will be given a sign. 
Fig. 8 Two representations of the same hook permutation $(H, \sigma)$. It has length 5 , size 23 , and content $(6,4,6,2,5)$.

Furthermore, $\sigma=(2,5,1,4,3)$, and $\sigma(H)$ has content $(6,6,5,2,4)$

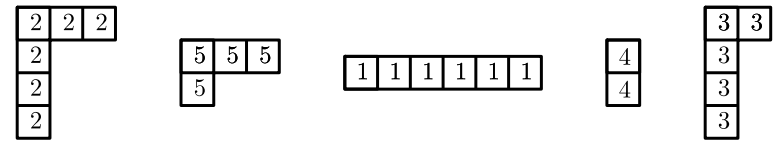

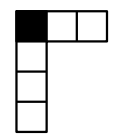
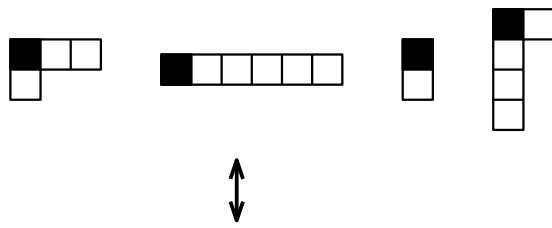

$\uparrow$

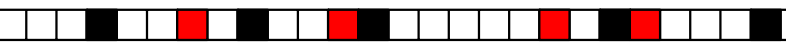

Fig. 9 The bijection in Proposition 4.1

\section{Local rules}

We wish to extend the local rules used by Shimozono and White [23] to deal with ribbons of all possible sizes; this will be done simply by reformulating White's insertion rules of [31] as local rules.

Throughout this section $\mu, v$ are two partitions of respective sizes $m$ and $n$.

For $i$ a nonnegative integer, we define $\mathcal{U}_{i}(\mu, \nu)$ as the set of partitions of size $\max (m, n)+i$ such that $\xi / \mu$ and $\xi / v$ are ribbons, or empty (this last case only appears for $i=0) . \mathcal{U}_{i}(\mu, \nu)$ is a signed set through $\operatorname{sgn}(\xi):=\varepsilon(\xi / \mu) \cdot \varepsilon(\xi / v)$. Similarly, we define $\mathcal{D}_{i}(\mu, \nu)$ the set of partitions of $\operatorname{size} \min (m, n)-i$ such that $\lambda / \mu$ and $\lambda / v$ are ribbons; $\mathcal{D}_{i}(\mu, \nu)$ is a signed set through $\operatorname{sgn}(\lambda):=\varepsilon(\mu / \lambda) \cdot \varepsilon(\nu / \lambda)$. In particular, $\mathcal{U}_{i}(\mu, \mu)$ and $\mathcal{D}_{i}(\mu, \mu)$ contain only positive elements. 
We draw a square where two corners are labeled by $\mu$ and $v$ as shown on the right. The bottom left corner will be labeled by partitions $\lambda$ from a set $\mathcal{D}_{i}(\mu, v)$, the top right one by partitions $\xi$ from a set $\mathcal{U}_{i}(\mu, \nu)$. In the case $\lambda=\mu=\nu$, the interior $C$ may be marked by a nonempty hook, or be left empty; in all other cases, it is left empty.

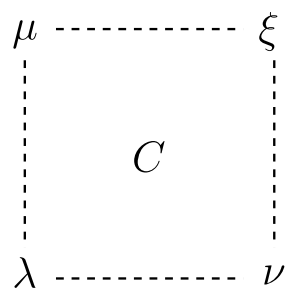

To define local rules, it is necessary to use the operations on ribbons and partitions defined in Sect. 2.

Let $((\lambda, C), \mu, v)$ be given in a square, as above. What we mean by applying $a$ local rule is the following: first finding out which case applies in the list below, then erasing $\lambda$ and $C$ from the square. Finally, rules D1 to D6 determine a partition $\xi$ that one writes in the top right corner, whereas rule $S$ determines a partition $\hat{\lambda}$ to be written in the bottom left corner.

Direct rules In the following rules, $\lambda$ is an element of a certain $D_{i}(\mu, v), C$ is empty unless possibly when $\lambda=\mu=v$ in which case it may be filled with a hook.

- If $\lambda=\mu=v$ and $C$ is empty, then $\xi:=\lambda$. (D1)

- If $\lambda=\mu=v$ and $C$ is a nonempty hook $E q$, then $\xi:=f i r s t(\lambda, E q)$. (D2)

- If $\lambda \neq \mu=v$, then $\xi:=\operatorname{next}(\mu, \mu / \lambda)$. (D3)

- If $\lambda=\mu \neq v$ (resp., $\lambda=v \neq \mu$ ), then $\xi:=v$ (resp., $\xi:=\mu$ ). (D4)

- If $\lambda, \mu, v$ are pairwise distinct partitions, let $r, r^{\prime}$ be the ribbons $\mu / \lambda$ and $\nu / \lambda$, and then:

- If $r$ and $r^{\prime}$ have neither the same tail nor the same head, then $\xi:=$ bumpout $\left(\lambda, r, r^{\prime}\right)$. (D5)

- If $r$ and $r^{\prime}$ have the same head but different tails, or the same tail but different heads, then:

$\triangleright$ If $\operatorname{slideout}\left(\lambda, r, r^{\prime}\right)$ is well defined, then $\xi:=\operatorname{slideout}\left(\lambda, r, r^{\prime}\right)$. (D6)

$\triangleright$ Otherwise, we set $\widehat{\lambda}:=\operatorname{switchout}\left(\lambda, r, r^{\prime}\right) \in D_{i}(\mu, v)$. (S)

Inverse rules Here $\xi$ belongs to a certain set $U_{i}(\mu, v)$. $C$ is left empty except in rule (I2).

- If $\xi=\mu=v$, then $\lambda:=\xi$. (I1)

- If $\xi \neq \mu=v$, then

- If $\operatorname{prev}(\xi, r)$ is not defined, we define $\lambda:=\mu$, and $C$ is filled with the unique hook of size $|r|$ and height $h(r)$; (I2)

- Otherwise $\lambda:=\operatorname{prev}(\xi, r)$. (I3)

- If $\xi=\mu \neq v$ (resp., $\xi=v \neq \mu$ ), then $\lambda:=v$ (resp., $\lambda:=\mu$ ). (I4)

- If $\xi, \mu, v$ are pairwise distinct partitions, let $r, r^{\prime}$ be the ribbons $\xi / \mu$ and $\xi / \nu$, and then:

- If $r$ and $r^{\prime}$ have neither the same tail nor the same head, then $\lambda:=$ $\operatorname{bumpin}\left(\xi, r, r^{\prime}\right)$. (I5)

- If $r$ and $r^{\prime}$ have the same head but different tails, or the same tail but different heads, then: 
$\triangleright$ If $\operatorname{slidein}\left(\xi, r, r^{\prime}\right)$ is defined, then $\lambda:=\operatorname{slidein}\left(\xi, r, r^{\prime}\right)$. (I6)

$\triangleright$ Otherwise we set $\widehat{\xi}:=\operatorname{switchin}\left(\xi, r, r^{\prime}\right) \in U_{i}(\mu, \nu)$. (T)

Proposition 5.1 The rules D1 to D6 are the respective inverses of $\mathrm{I} 1$ to I6; S and T are involutions. Furthermore, D1-D6 and I1-I6 preserve signs between $\mathcal{D}_{i}(\mu, v)$ and $\mathcal{U}_{i}(\mu, v)$, whereas $\mathrm{S}$ and $\mathrm{T}$ are sign reversing on $\mathcal{D}_{i}(\mu, v)$ and $\mathcal{U}_{i}(\mu, \nu)$, respectively.

Proof All these properties are already proved elsewhere, albeit sometimes in a different form. For D2, D3 and I2, I3, this was proved by Shimozono and White [23]. For the rules D5, D6, I5, I6, S, and T, the result can be found in White [31]. We will nevertheless give the proof for the rule $\mathrm{S}$ in Appendix, using an encoding of partitions by infinite sequences; we wish to show how this encoding is particularly suited to the study of ribbons.

Let us sum up the local rules in terms of signed bijections, since this will be useful in particular in the algebraic approach of Sect. 9:

Proposition 5.2 Let $\mu, v$ be two partitions and $i$ a positive integer.

(a) There exists a bijection $\phi_{1}$ between $\mathcal{U}_{i}(\mu, \mu)$ and $\mathcal{D}_{i}(\mu, \mu) \sqcup \llbracket 0, i-1 \rrbracket$.

(b) For $\mu \neq v$, there exists a signed bijection $\left(i_{D}, i_{U}, \phi_{2}\right)$ between $\mathcal{D}_{i}(\mu, v)$ and $\mathcal{U}_{i}(\mu, v)$.

Proof (a) Such a bijection is given by rules D2 and D3; for (b), the involutions $i_{D}$ and $i_{U}$ are given by rules $S$ and T, respectively, and the bijection $\phi_{2}$ consists of rules D4, D5, and D6.

This proposition has to be interpreted as a local property of the graph GR: given $\mu$ and $v$ of size $m$ and $n$, it gives a combinatorial link between vertices adjacent to both $\mu$ and $v$ at the level $\min (m, n)-i$, and at the level $\max (m, n)-i$. Sections 6 and 9 will use this local structure to deduce global results on ribbon tableaux.

\section{Bijective approach}

We want to use growth diagram techniques (see [7]) to carry out a signed correspondence proving Theorems 7.1 and 7.2. This will be done in this section, but it requires more work than a simple application of Fomin's setting. In order to prove Theorem 7.1, we will in particular need to make some back and forth moves in a growth diagram; the correctness of the correspondence will rely on the involution principle.

\subsection{Hook permutations and pairs of ribbon tableaux}

Fix a positive integer $\ell$, and let $G_{\ell}$ be the grid of size $\ell \times \ell$, made of $\ell^{2}$ squares (and $(\ell+1)^{2}$ vertices). Square $(i, j)$ is at the intersection of the $i$ th row from the bottom and the $j$ th column from the left. We wish now to label some of the vertices 
by partitions and then apply the local rules of Sect. 5 in the squares; for this, we order the squares (partially) by $(i, j) \preceq\left(i^{\prime}, j^{\prime}\right)$ if and only if $i \leq i^{\prime}$ and $j \leq j^{\prime}$.

We fix from now on a total order $\mathcal{O}$ extending this partial order. Every square $s q \neq$ $(1,1)$ has then a predecessor $\operatorname{pred}(s q)$, and every square $s q \neq(\ell, \ell)$ has a successor $\operatorname{succ}(s q)$. For $\operatorname{dir}= \pm 1$, we also define $\operatorname{Next}(s q, \operatorname{dir})$ to be $\operatorname{succ}(s q)$ if $\operatorname{dir}=1$ and $\operatorname{pred}(s q)$ if $\operatorname{dir}=-1$, and to return "undefined" when pred or succ is not defined.

Given $\operatorname{dir} \in\{+1,-1\}$ and a square $s q$ of $G_{\ell}$ :

- If $\operatorname{dir}=1$ and $\mu, v, \lambda, C$ label $s q$ as in the definition of direct rules, we apply the corresponding rule;

- If $\operatorname{dir}=-1$ and $\mu, v, \xi$ label $s q$ as in the definition of inverse rules, we apply the corresponding rule.

Let us call this procedure Apply_local_rule; if loc is the local rule that applies, we write $l o c:=$ Apply_local_rule $($ dir,$s q)$.

Now we want to go from local rules to a correspondence on the entire grid. Let us be given a hook permutation drawn on $G_{\ell}$, in which we also label by $\varnothing$ (the empty partition) all vertices of $G_{\ell}$ on the bottom and left sides (see configuration A on Fig. 10). We may now describe the bijection $\phi$ of Theorem 7.1, which we do in an algorithmic fashion (a precise definition of $\phi$ will be given in Sect. 8).

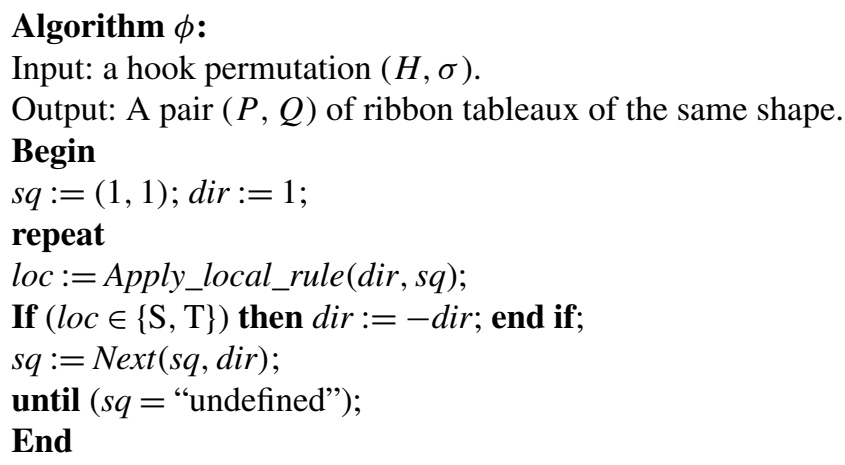

We will show in Sect. 8 that this algorithm is well defined and does not loop indefinitely; it ends when $\operatorname{succ}(\ell, \ell)$ is not defined, in which case the vertices on the top and right side of $G_{\ell}$ are labeled by partitions forming two ribbon tableaux of the same shape.

We can also consider the opposite procedure, starting this time with the input of a pair of ribbon tableaux of the same shape, and initializing the procedure in the square $(\ell, \ell)$ with $d i r$ equal to -1 . In this case, it turns out that the procedure ends either with $\operatorname{pred}(1,1)$ or $\operatorname{succ}(\ell, \ell)$ undefined; in the former case, it is an inverse to the procedure $\phi$, while in the latter it defines an involution $i$ on pairs of ribbon tableaux of the same shape.

Example We illustrate the algorithm on the example of Fig. 10. For the total order $\mathcal{O}$, we choose the column $\operatorname{order}(i, j)<\left(i^{\prime}, j^{\prime}\right)$ if $j<j^{\prime}$, or $j=j^{\prime}$ and $i<i^{\prime}$. A square $c^{\prime}$ is thus larger than a square $c$ if it is above in the same column, or if $c^{\prime}$ is in a column to the right of $c$. 

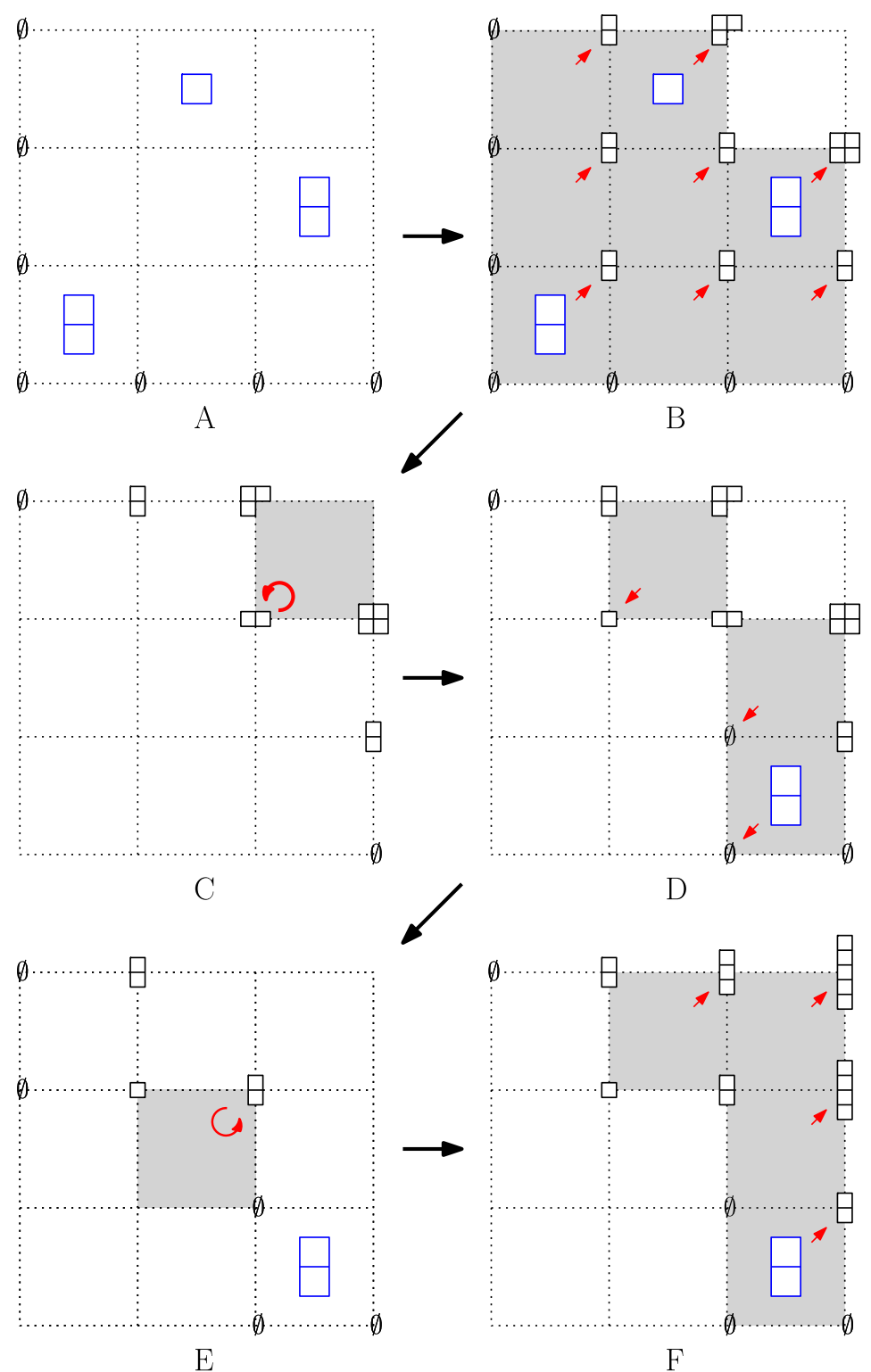

$\mathrm{D}$

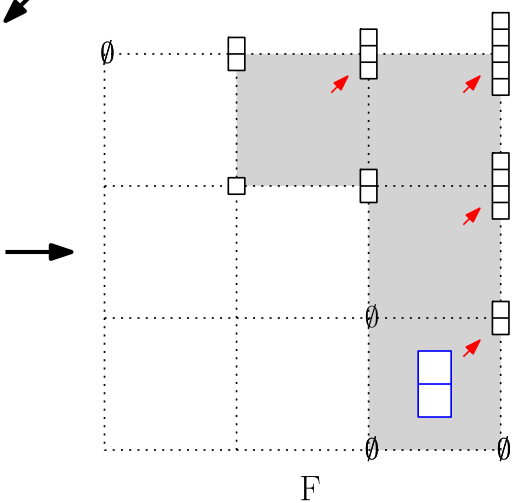

Fig. 10 The Algorithm $\phi$ with column order

We start with the hook permutation given on $G_{3}$ (configuration A). We apply direct local rules to reach configuration B, the rules being successively D2, D4, D4, D4, D1, D2, D4, and D2. Now rule S applies, and the direction changes (configuration C). Note that we deleted all contents of visited squares as well as the label of their bottom left corner; we did not show this for direct rules in order to keep the number of pictures reasonable. From this configuration C, we apply inverse rules I5, I2, and I5 

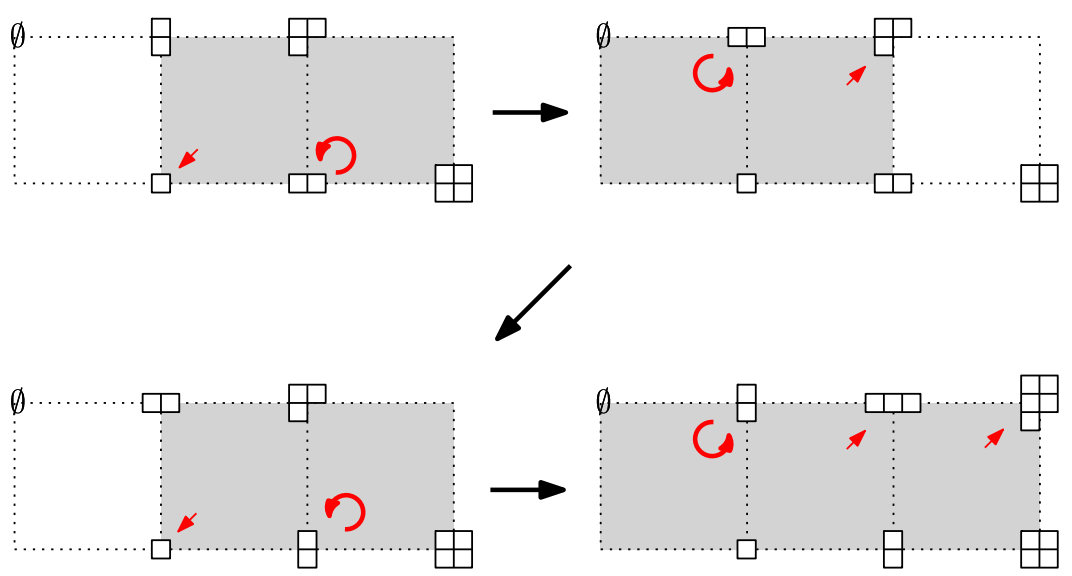

Fig. 11 The Algorithm $\phi$ with row order. We only represent the upper part of the growth diagram, and start after the case B of Fig. 10 which is also obtained with the row order

successively to reach configuration $\mathrm{D}$. There rule $\mathrm{T}$ applies, and the direction changes. We finally reach configuration $F$ with the rules D3, D2, D3, and D6. We can finally read off the ribbon tableaux $P$ and $Q$, respectively, on the right and top sides of the grid.

Remark 6.1 The choice of the order $\mathcal{O}$ matters; that is, changing the order can result in a different correspondence. For instance, if the row order is chosen in the example of Fig. 10, then the given hook permutation will be sent to another pair of tableaux, as illustrated in Fig. 11.

A special case of this is that if, for a given order $\mathcal{O},(P, Q)$ is associated to a hook permutation $(H, \sigma)$, then $(Q, P)$ is not necessarily equal to the inverse hook permutation. Indeed, by transposition, the example of Fig. 11 can be interpreted as computing the pair of ribbon tableaux associated to the inverse of the hook permutation of Fig. 10.

\subsection{Hook involutions and ribbon tableaux}

\subsubsection{Hook involutions}

Definition 6.1 A hook involution is a hook permutation $(H, \sigma)$ such that $\sigma=\sigma^{-1}$ and $H_{i}=H_{\sigma(i)}$ for all $i$.

In the array representation, hook involutions are the hook permutations such that a cell and its symmetric with respect to the diagonal $i=j$ have the same content. For a hook involution $I=(H, \sigma)$, we define its sign as $\varepsilon(I)=\prod_{i / \sigma(i)=i} \varepsilon\left(H_{i}\right)$. It is the product of the signs of the hooks associated to fixed points. We denote by $\mathcal{H} \mathcal{I}$ the signed set of hook involutions, by $\mathcal{H} \mathcal{I}(\mu)$ the signed subset of those hook involutions with content $\mu$. 
Finally, for use in Sect. 10 only, we define $\mathcal{H} \mathcal{I}_{\text {spec }}(\mu)$ the subset of $\mathcal{H} \mathcal{I}(\mu)$ consisting of those hook involutions $(H, \sigma)$ which verify that for any fixed point $i=\sigma(i)$, the hook $H_{i}$ has height 0 and odd size. Note that all elements of $\mathcal{H} \mathcal{I}_{\text {spec }}(\mu)$ are positive.

Lemma 6.1 For any composition $\mu$ there holds $\left|\mathcal{H} \mathcal{I}(\mu) \backslash \mathcal{H} \mathcal{I}_{\text {spec }}(\mu)\right|_{ \pm}=0$.

Proof Let $I=(H, \sigma)$ be an element of $\mathcal{H} \mathcal{I}(\mu) \backslash \mathcal{H} \mathcal{I}_{\text {spec }}(\mu)$. We define $i=\sigma(i)$ to be the smallest fixed point whose hook $H_{i}$ has even size or nonzero height, and we let $h, s$ be respectively the height and size of $H_{i}$. If $s$ is even, then we let $H_{i}^{\prime}$ be the hook of size $s$ and of height $h+1$ (resp., $h-1$ ) if $h$ is even (resp., odd); if $s$ is odd, then $h \neq 0$ necessarily, and we let $H_{i}^{\prime}$ be the hook of size $s$ and height $h+1$ (resp., $h-1$ ) if $h$ is odd (resp., even).

Now let $H^{\prime}$ be the hook list equal to $H$ except in position $i$ where $H_{i}^{\prime}$ replaces $H_{i}$. If we define $f(I):=\left(H^{\prime}, \sigma\right)$, then it is readily verified that $f$ is a sign reversing involution on $\mathcal{H} \mathcal{I}(\mu) \backslash \mathcal{H} \mathcal{I}_{\text {spec }}(\mu)$, and the lemma is proved.

Corollary 6.1 For any composition $\mu,|\mathcal{H} \mathcal{I}(\mu)|_{ \pm}=\left|\mathcal{H} \mathcal{I}_{\text {spec }}(\mu)\right|$.

We will give some consequences of this result in Sect. 10.

\subsubsection{Signed correspondence with ribbon tableaux}

The Schensted correspondence is known to induce a bijection between involutions and standard tableaux. We wish to generalize this bijection to hook involutions and ribbon tableaux. But whereas, in the Schensted correspondence, one simply needs a restriction, some extra work has to be done here: first, the bijection defined by the algorithmic procedure depends on the fixed total order specified on the squares of the grid, so the procedure cannot be done symmetrically in general. The modification is simple: one works only with the squares $(i, j)$ of the grid verifying $i \geq j$, which are those weakly to the north west of the diagonal. The algorithmic procedure works then the same way, by performing local rules according to a given fixed order on the cells; ribbon tableaux are read off on the upper boundary of the grid. Here however, when starting with a hook involution, one may finish with another hook involution of a different sign; this is the second point which differs from the case of hook permutations which were defined as positive. We will show how to deal with these two extra difficulties, in order to obtain Theorem 7.2.

To finish, we formulate in terms of signed sets the properties which turn out to be essential in the proof of Theorem 7.2. Define $\mathcal{U}_{i}(\mu)$ as the set $\mathcal{U}_{i}(\mu, \mu)$ but with sign $\varepsilon(\lambda / \mu)$ for $\lambda \in \mathcal{U}_{i}(\mu)$; similarly, $\mathcal{D}_{i}(\mu)$ is $\mathcal{D}_{i}(\mu, \mu)$ with sign $\varepsilon(\mu / \lambda)$.

Proposition 6.1 Consider $\llbracket 0, i-1 \rrbracket$ as a signed set with $\operatorname{sgn}(h)=(-1)^{h}$. Then there is a sign preserving bijection between $\mathcal{U}_{i}(\mu)$ and $\mathcal{D}_{i}(\mu) \sqcup \llbracket 0, i-1 \rrbracket$.

This is an immediate consequence of Proposition 2.1. 


\section{Main results}

The first result is a generalization of the Schensted correspondence. We denote by Id the identity function on hook permutations.

Theorem 7.1 (White [31, Theorem 6]) Let $n, \ell$ be two positive integers. The data (Id, $i, \phi)$ is a signed bijection between (i) hook permutations of size $n$ and length $\ell$ and (ii) pairs of ribbon tableaux of size $n$ and length $\ell$ with the same shape.

This bijection preserves contents in the sense that if $i(P, Q)=\left(P_{1}, Q_{1}\right)$, then $c(P)=c\left(P_{1}\right)$ and $c(Q)=c\left(Q_{1}\right)$; and if $\phi(H, \sigma)=(P, Q)$, then $c(H)=c(Q)$ and $c(\sigma(H))=c(P)$.

The bijections were defined in Sect. 6; the proof of the correctness of the bijection is deferred to Sect. 8 .

The correspondence of the theorem is described by [31] with an involved insertion algorithm which generalizes the one in the Schensted correspondence. Also, White also assumes that the contents of $P$ and $Q$ verify a certain compatibility relation, but remarks at the end of his paper that this condition may be dropped.

As shown in Sect. 6, our idea here was to use Fomin's techniques [6, 7] in the proof of this result. This sheds a new light on White's result, and incidentally lends itself to generalization in a more straightforward fashion, as we will point out in Sect. 11.

The theorem has the following consequences concerning the signed enumeration of ribbon tableaux:

Corollary 7.1 Let $\mu, v$ be two compositions of $n$ with $\ell$ parts, and write $\tilde{\mu}=$ $\left[1^{j_{1}}, 2^{j_{2}}, \ldots\right]$. Then

$$
\begin{aligned}
\sum_{\substack{\lambda \in \mathbf{Y}_{n} \\
P \in R T_{\lambda, \mu}, Q \in R T_{\lambda, v}}} \varepsilon(P) \varepsilon(Q) & =\delta_{\widetilde{\mu} \widetilde{v}} \cdot 1^{j_{1}}\left(j_{1} !\right) 2^{j_{2}}\left(j_{2} !\right) \cdots ; \\
\sum_{\substack{\lambda \in \mathbf{Y}_{n} \\
P, Q \in R T_{\lambda}(\ell)}} \varepsilon(P) \varepsilon(Q) & =\left(\begin{array}{c}
n+\ell-1 \\
2 \ell-1
\end{array}\right) \cdot \ell !
\end{aligned}
$$

We will prove the correctness of Theorem 7.1 in Sect. 8, but we can already give the proof of its corollary:

Proof of Corollary 7.1 For the first formula, the signed bijection of Theorem 7.1 implies that the left-hand side is equal to the number of hook permutations of type $\left(c, c^{\prime}\right)$; this number is zero unless $c$ can be obtained from $c^{\prime}$ by permuting some of its parts. When $\widetilde{c}=\widetilde{c}^{\prime}$, we have to compute, for a part $i$ appearing $j_{i}$ times, how many hook permutations of length $j_{i}$ exist so that all hooks have size $i$; this number is clearly $i^{j_{i}} j_{i}$ !. We obtain finally the right-hand side by multiplying such terms for all sizes $i$. The second equality of Corollary 7.1 is an immediate consequence of Theorem 7.1 and of Proposition 4.1. 
We will deduce easily this corollary from the theorem in Sect. 6, and give alternative proofs using linear algebra in Sect. 9. Note that the first part of this corollary was the focus of [31].

Finally, the Schensted correspondence has the property that it restricts to a bijection between involutions of $\mathfrak{S}_{n}$ and standard tableaux of size $n$. We have a counterpart of this result for general ribbon tableaux, thanks to the procedure described in Sect. 6.2:

Theorem 7.2 There exists a content-preserving signed bijection between hook involutions and ribbon tableaux.

As explained in Sect. 6, this cannot be deduced from White's correspondence for pairs of tableaux. We will prove this Theorem in Sect. 8. We also give an enumerative consequence of this theorem in Sect. 10 - this is Theorem 10.2, of which we also give both a bijective proof and an algebraic one.

Relation with previous work As already mentioned, Theorem 7.1 was essentially demonstrated by White [31] (in this article, White evokes the possibility of extending his ideas to obtain the form in which we gave it).

In [28], the authors notice that if one only considers hook permutations with all hooks of size $k$, and ribbon tableaux with all ribbons of size $k$, then rules $\mathrm{S}$ and $\mathrm{T}$ can never be applied: so in this case we have a (sign-preserving) bijection between ribbon tableaux and $k$-colored permutations.

As a matter of fact, rules D2 and D3 are not used in these two articles, but alternative rules that do not preserve signs in the sense of Proposition 6.1. Theorem 7.2 cannot thus be a consequence of White's original work, but is based on the work of Shimozono and White [23] (for ribbons and hooks of fixed size), in which the authors introduce the operations prev, next, first that are used to define rules D2, D3 and I2, I3.

\section{Proof of Theorems 7.1 and 7.2}

In this section, we will give the proofs of Theorems 7.1 and 7.2, the signed bijections having been defined in Sect. 6. The proof is directly inspired by Fomin's constructions [7], but some extra technicalities are needed in both proofs.

\subsection{Proof of Theorem 7.1}

We show that the construction $\phi$ defined algorithmically in Sect. 6.1 verifies indeed all properties stated in the theorem. We will demonstrate that this algorithm is, in fact, a consequence of Garsia and Milne's involution principle. Therefore, we have to construct signed sets $\mathcal{A}, \mathcal{B}$ and adequate functions as explained in Sect. 3. These signed sets will be called configurations, and defining them rigorously requires the introduction a certain number of concepts. 
We call a cutting path of the grid $G_{\ell}$ a path from the top left vertex to the bottom right one, with south and east steps. We call inside of the cutting path $F$ the squares of $G_{\ell}$ to the south west of $F$, and outside the rest of the squares.

A good labeling of a cutting path $F$ is a labeling of each of its vertices by a partition such that:

- The vertices at the top left and bottom right are labeled by the empty partition $\emptyset$.

- For every horizontal edge of $F$, the labels $\lambda$ and $\mu$ at the left and right end respectively form a ribbon $\mu / \lambda$.

- For every vertical edge of $F$, the labels $\lambda$ and $\mu$ at the bottom and top end respectively form a ribbon $\mu / \lambda$.

We allow $\lambda=\mu$ in this definition, corresponding to the empty ribbon. Now we fixed a total order $\mathcal{O}$ on the squares of $G_{\ell}$ in Sect. 6; an $\mathcal{O}$-cutting path is a cutting path $F$ such that the squares inside $F$ are smaller than the squares outside. Such a cutting path defines in particular two squares in general: $s q_{<}(F)$ which is the largest square inside $F$, and $s q_{>}(F)$ the smallest one outside. Clearly, $s q_{<}(F)$ is not defined when $F$ consists of the left and bottom side of the grid, and $s q_{>}(F)$ is not defined when $F$ consists of the top and right side of the grid.

Let $F$ be a cutting path with a good labeling label, which induces a labeling of the edges of $F$ by ribbons. We define a coloring as a partial filling by nonempty hooks of the squares of $G_{\ell}$, and such a coloring col is compatible with $(F$, label $)$ if:

- The squares inside $F$ are not filled.

- For every horizontal edge $h$ of $F$ labeled by $r$, there is exactly one square filled by a hook in the column above $h$ when $r$ is empty, and none if $r$ is nonempty.

- For every vertical edge $v$ of $F$ labeled by $r$, there is exactly one square filled by a hook in the row right of $v$ when $r$ is empty, and none if $r$ is nonempty.

These labeled cutting paths, together with a coloring, model what the intermediate objects are in the application of Algorithm $\phi$ in Sect. 6.

Configurations We can now introduce configurations which are the main objects we will consider for the rest of the proof.

Definition 8.1 A configuration is a 3-tuple ( $F$, label, col) where $F$ is an $\mathcal{O}$-cutting pathwhich is well labeled by label, and col is a coloring of $G_{\ell}$ compatible with $(F$, label).

Let $(F$, label, col $)$ be a configuration on $G_{\ell}$. For each $i \in \llbracket 1, \ell \rrbracket$, we denote by $c_{i}>0$ the size of the hook in column $i$, or the size of the ribbon labeling the edge of $F$ appearing in column $i$; by compatibility of label and col, exactly one of these two cases occur. Likewise, we denote by $c_{i}^{\prime}$ the size of the hook in row $i$, or the size of the ribbon labeling the edge of $F$ appearing row $i$. The content of a configuration is then defined as the two compositions $\left(c, c^{\prime}\right)$, where $c=\left(c_{1}, \ldots, c_{\ell}\right)$ and $c^{\prime}=\left(c_{1}^{\prime}, \ldots, c_{\ell}^{\prime}\right)$.

Let us define the sign of a configuration $(F$, label, col $)$ as the product of all $2 \ell$ ribbons labeling the edges of $F$ (recall that the empty ribbon has sign +1 ). For instance, the steps $\mathrm{C}$ and $\mathrm{E}$ of Fig. 10 show two configurations: $\mathrm{C}$ and $\mathrm{E}$ have both content $((2,1,2),(2,2,1))$, and $C$ has negative sign, whereas $\mathrm{E}$ has positive sign. 
So configurations are signed objects to which a certain content is attached, and we can view the objects involved in Theorem 7.1 as special subclasses of permutations, as we now show.

A hook permutation $(H, \sigma)$ is naturally a positive configuration: the border is the left and bottom side of $G_{\ell}$, all vertices are labeled by $\varnothing$, and the coloring is just the representation of $(H, \sigma)$ in the grid. The content of such a configuration is $(c(H), c(\sigma(H)))$. A pair $(P, Q)$ of ribbon tableaux of length $\ell$ with the same shape is also a configuration: the border is the top and right side, the right side being labeled by $P$ and the top side by $Q$; and all squares of the grid are empty. The sign of the configuration is $\varepsilon(P) \varepsilon(Q)$, and the content is $(c(Q), c(P))$; note the swap of the tableaux here. We will from now on identify hook permutations and pairs of ribbon tableaux to such configurations.

We now notice, in the algorithm describing our correspondence, the transformation Apply_local_rule on pairs consisting of a configuration together with a direction. An inspection of each local rule shows that the compatibility conditions in Definition 8.1 are indeed respected by this transformation. Furthermore, the content of a configuration is invariant by this transformation.

Application of the involution principle of Garsia and Milne We now fix two compositions $c_{1}$ and $c_{2}$ of length $\ell$ and size $n$, and consider from now on only configurations with content $\left(c_{1}, c_{2}\right)$.

A configuration $(F$, label, col) is said to be of type $A$ if rule $\mathrm{T}$ is the rule to be applied in $s q_{<}(F)$, or if it is a permutation (which is when $s q_{<}(F)$ is not defined). It is of type $B$ if rule $\mathrm{S}$ has to be applied in $s q_{>}(F)$, or if it is a pair of ribbon tableaux (which is when $s q_{>}(F)$ is not defined). Let $\mathcal{A}$ (resp., $\mathcal{B}$ ) be the set of configurations of type $\mathrm{A}$ (resp., B). For instance, the configurations $\mathrm{C}$ and $\mathrm{E}$ of Fig. 10 are of type $\mathrm{A}$ and $\mathrm{B}$, respectively, for the content $((2,1,2),(2,2,1))$. Note that configurations can happen to be of both types.

Now we define the bijection and the two involutions that are involved in the application of the involution principle. First, define an involution on $\mathcal{A}$ by applying the rule $\mathrm{T}$ in $s q_{<}(F)$ when it is defined, and keeping the hook permutations unchanged. Likewise, we have an involution on $\mathcal{B}$ by applying the rule $S$ in $s q_{>}(F)$ when it is defined, and letting the pairs of tableaux unchanged. Notice that these two involutions are sign reversing by Proposition 5.1. We define also a bijection $\mathcal{A} \rightarrow \mathcal{B}$ by applying direct rules in $s q_{>}(F)$ on a type A configuration, until we reach a type B configuration. Since in this case we only apply rules of the form $\mathrm{D} i$, the sign is preserved, thanks to Proposition 5.1 again. The inverse bijection from $\mathcal{B}$ to $\mathcal{A}$ goes simply by applying repeatedly inverse rules in $s q_{<}(F)$ on a type B configuration, until we reach a type A configuration.

We now have all the necessary functions, and the application of the involution principle gives us a signed bijection between hook permutations and pairs of ribbon tableaux that verifies exactly the properties of Theorem 7.1. This is precisely the correspondence defined in Sect. 6, and completes the proof.

\subsection{Proof of Theorem 7.2}

We will use here the definitions used in the proof of Theorem 7.1, and adapt them to the case of involutions. We consider a total order $\mathcal{H O}$ on $H G_{\ell}$, the half grid 
Fig. 12 A half configuration

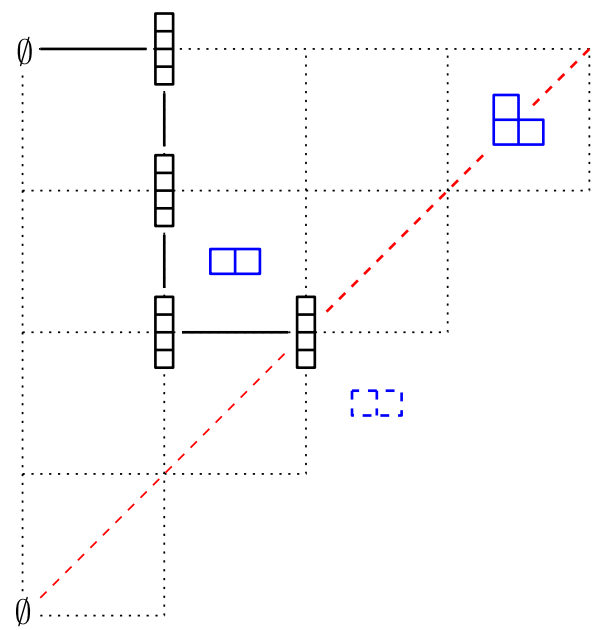

made of the squares $(i, j)$ of $G_{\ell}$ with $i \geq j$, and suppose that this order extends the order $\preceq$, defined by $(i, j) \preceq\left(i^{\prime}, j^{\prime}\right)$ if $i \leq i^{\prime}$ and $j \leq j^{\prime}$. This induces a partial order on the squares of $G_{\ell}$, given by: $(i, j)$ is smaller than $\left(i^{\prime}, j^{\prime}\right)$ if and only if $(\min (i, j), \max (i, j))$ is smaller than $\left(\min \left(i^{\prime}, j^{\prime}\right), \max \left(i^{\prime}, j^{\prime}\right)\right)$ for $\mathcal{H O}$; in this way a square and its symmetric are not comparable. We will still denote this partial order by $\mathcal{H O}$.

We consider configurations as in Definition 8.1, with the exception that the order $\mathcal{H O}$ is now used. A configuration ( $F$, label, col) on $G_{\ell}$ is called symmetric if $F$, label, and col are all symmetric with respect to the diagonal $i=j$; we define a half-configuration as the restriction to $H G_{\ell}$ of a symmetric configuration. Note that the border $f$ of a half-configuration consists of a path form the top left corner of $H G_{\ell}$ to any vertex of the diagonal $i=j$. Figure 12 shows an example of a half configuration.

For a half configuration, applying a local rule in the square $(i, j) \in H G_{\ell}$ means applying it in both $(i, j)$ and $(j, i)$ in the associated symmetric configuration, and restricting the result to $H G_{\ell}$. Note that local rules in $(i, j)$ and $(j, i)$ will give the same outputs since all our local rules are symmetric in $\mu$ and $\nu$, so that we will only deal with symmetric configurations.

We represent naturally ribbon tableaux by a chain of partitions on the top side of $H G_{\ell}$, and hook involutions by the restriction of their matrix representation to $H G_{\ell}$ with $\varnothing$ labeling the vertices on the left. The content $c$ of a symmetric configuration is of the form $(c, c)$, so we define the content of the associated half configuration as $c$. The sign of a half configuration $(f$, label, $c o l)$ is the product of all the signs labeling $f$, multiplied by the product of the signs of hooks appearing in the square $(i, i)$. Note that this gives the desired sign on $\mathcal{H} \mathcal{I}$ and on ribbon tableaux, so that we are indeed in the setting of Theorem 7.2.

Let us denote by $\mathcal{H} \mathcal{A}$ and $\mathcal{H B}$ the sets of half configurations with associated symmetric configurations in $\mathcal{A}$ and $\mathcal{B}$, respectively, with the partial order $\mathcal{H O}$. We define the involutions on $\mathcal{H} \mathcal{A}$ and $\mathcal{H B}$ in the same fashion as for $\mathcal{A}$ and $\mathcal{B}$, as well as the bijection between $\mathcal{H} \mathcal{A}$ and $\mathcal{H} \mathcal{B}$. 
Now we wish to apply the involution principle with these two involutions and this bijection: we have to check the sign modifications here since the definition of the sign of a configuration has been modified. For the non-diagonal squares $(i, j)$ with $i>j$, everything works as before: the application of a rule $\mathrm{S}$ or $\mathrm{T}$ changes the sign, whereas the other rules preserve it. Therefore, the two involutions are indeed sign-reversing, and the bijection is sign-preserving.

There remains the application of a rule on a diagonal square $s q$. First, we notice that rules D1-D3 and I1-I3 are the only ones that can be applied there since they are the only rules with $\mu=v$. So what we finally have to prove is that the sign of the ribbon on the left side of $s q$, times the sign of the hook in $s q$ in the case of D2, is equal to the sign of the ribbon on the top side of $s q$.

This is trivial for the rule D1. For the rules D2 and D3, one has to look at the definition of prev and first (Sect. 2.2): $\operatorname{first}(\lambda, e q)$ is of the same height as eq by definition, and thus of the same sign: this implies that rule D2 will indeed have the sign preserving property. For the rule D3, $\operatorname{next}(\mu, \mu / \lambda)$ is a ribbon of the same height as $\mu / \lambda$, and thus of the same sign, which implies here also that the rule preserves the sign. The involution principle can thus be applied, and this achieves the proof of Theorem 7.2.

The end of the proof shows why this cannot be deduced from White's work [31]: the rules he used in the case $\mu=v$ do not preserve the signs we use here, which are the ones discovered only much later in [23].

\section{Algebraic approach}

The previous section generalized the Robinson-Schensted bijection. Now we will give an algebraic proof of the enumerative consequence of these results, that is, Corollary 7.1, in the spirit of Stanley [25] and Fomin [6].

Let $\mathbb{K}$ be a field of characteristic zero. We consider $\mathbb{K} \mathbf{Y}=\bigoplus_{n} \mathbb{K} \mathbf{Y}_{n}$, the vector space of all (finite) linear combinations of partitions with coefficients in $\mathbb{K}$. For $i$ a positive integer, we define two linear operators $U_{i}$ and $D_{i}$ via their action on the basis of partitions.

Definition 9.1 For $\lambda \in \mathbf{Y}$,

$$
U_{i} \lambda=\sum_{r=\mu / \lambda \in R i b_{i}} \varepsilon(r) \mu ; \quad D_{i} \lambda=\sum_{r=\lambda / \mu \in R i b_{i}} \varepsilon(r) \mu .
$$

$U_{i}$ and $D_{i}$ are endomorphisms of $\mathbb{K} \mathbf{Y}$ that send $\mathbb{K} \mathbf{Y}_{n}$ into $\mathbb{K} \mathbf{Y}_{n+i}$ and $\mathbb{K} \mathbf{Y}_{n-i}$, respectively. We note that these two operators were already defined by Stanley [25] but with a different perspective.

For $\lambda, \mu$ two partitions, we set $\langle\lambda, \mu\rangle=1$ if $\lambda=\mu$ and 0 otherwise. We may then extend $\langle\cdot, \cdot\rangle$ to $\mathbb{K} \mathbf{Y} \times \mathbb{K} \mathbf{Y}$ by bilinearity. Notice that $U_{i}$ and $D_{i}$ are dual endomorphisms for this bilinear form. Indeed $\left\langle U_{i} \lambda, \mu\right\rangle=\left\langle\lambda, D_{i} \mu\right\rangle$ for any $\lambda, \mu$, since each side is equal to $\varepsilon(r)$ if $\lambda \subseteq \mu$ and $r=\mu / \lambda$ is a ribbon, and to 0 otherwise.

The fundamental relations between these endomorphisms provides the following (we denote by $A B=A \circ B$ the composition): 
Proposition 9.1 For nonnegative integers $i, j$, we have

$$
\begin{aligned}
& D_{i} U_{i}=U_{i} D_{i}+i \cdot \mathrm{Id}, \\
& D_{i} U_{j}=U_{j} D_{i} \quad \text { if } i \neq j .
\end{aligned}
$$

Proof The first equality can be rewritten

$$
\begin{aligned}
\left.\qquad D_{i} U_{i} \mu, v\right\rangle & =\left\langle U_{i} D_{i}+i \cdot \operatorname{Id} \mu, v\right\rangle, \\
\text { or equivalently, }\left\langle D_{i} U_{i} \mu, v\right\rangle & =\left\langle U_{i} D_{i} \mu, v\right\rangle+i \cdot \delta_{\mu, \nu}, \\
\text { or equivalently, }\left\langle U_{i} \mu, U_{i} v\right\rangle & =\left\langle D_{i} \mu, D_{i} v\right\rangle+i \cdot \delta_{\mu, v}
\end{aligned}
$$

for $\mu$ and $\nu$ any two partitions, while the second equality is equivalent to

$$
\left\langle D_{i} U_{j} \mu, v\right\rangle=\left\langle U_{j} D_{i} \mu, v\right\rangle .
$$

Those two equalities can be rephrased as $\left|\mathcal{U}_{i}(\mu, \mu)\right|=\left|\mathcal{D}_{i}(\mu, \mu)\right|+i$ for all $\mu$, and $\left|\mathcal{U}_{i}(\mu, v)\right|_{ \pm}=\left|\mathcal{D}_{i}(\mu, v)\right|_{ \pm}$for $\mu \neq v$. But this is exactly the enumerative signification of Proposition 5.2, which ends the proof.

The first of these two relations is characteristic of $i$-differential posets, defined by Stanley $[25,26]$, and which are the basis of [6].

Now we consider infinite matrices with coefficients in $\mathbb{K}:=\mathbb{Q} \llbracket q \rrbracket$, where rows and columns are indexed by $\mathbf{Y}$; in particular, $D_{i}$ and $U_{i}$ are now considered as such matrices. We will write vectors as infinite linear combinations of partitions with coefficients in $\mathbb{K}$. Let us now define our main matrices $\mathbf{U}$ and $\mathbf{D}$ :

\section{Definition 9.2}

$$
\mathbf{U}=\sum_{i} q^{i} U_{i} ; \quad \mathbf{D}=\sum_{j} q^{j} D_{j} .
$$

$\mathbf{U}_{\lambda, \mu}$ (resp., $\mathbf{D}_{\lambda, \mu}$ ) is thus equal to $\varepsilon q^{k}$ if $\mu / \lambda$ (resp., $\lambda / \mu$ ) is a ribbon of size $k$ and sign $\varepsilon$, and 0 otherwise. This interpretation gives us immediately the following formula:

$$
\sum_{\substack{\lambda \in \mathbf{Y}_{n} \\ P, Q \in R T_{\lambda, \ell}}} \varepsilon(P) \varepsilon(Q)=\left[q^{2 n}\right]\left(\mathbf{D}^{\ell} \mathbf{U}^{\ell}\right)_{\emptyset, \emptyset} .
$$

We want to calculate the right-hand side of this equality; note first that Proposition 9.1 can be summarized in a single relation involving $\mathbf{U}$ and $\mathbf{D}$ :

$$
\mathbf{D U}=\mathbf{U D}+\frac{q^{2}}{\left(1-q^{2}\right)^{2}} \mathrm{Id} .
$$

Proof One just has to take the definition of $\mathbf{U}$ and $\mathbf{D}$, develop, and then use the relations of Proposition 9.1. The coefficient of Id appearing is then $\sum_{i \geq 1} i q^{2 i}$, which is equal to the given rational fraction. 
In order to compute the coefficient of $q$ in (5), we need the following result of Stanley:

Theorem 9.1 (Stanley [25]) Let two matrices $D, U$ verify $D U=U D+r I$ for a certain element $r$. Then for all positive integers $\ell$ we have

$$
D^{\ell} U^{\ell}=(U D+r I)(U D+2 r I) \cdots(U D+\ell r I) .
$$

Thus if $\widehat{O}$ is a vector such that $D \widehat{O}=0$, we have $\left(D^{\ell} U^{\ell}\right) \widehat{O} \widehat{O}=r^{\ell} \ell$ !

By (6), $\mathbf{U}$ and $\mathbf{D}$ verify the condition of the theorem with $\widehat{O}=\emptyset$ and $r=q^{2} /$ $\left(1-q^{2}\right)^{2}$. Therefore, the second formula of Corollary 7.1 follows from (5), Theorem 9.1, and the following computation:

$$
r^{\ell} \ell !=\ell ! \cdot q^{2 \ell} \cdot \frac{1}{\left(1-q^{2}\right)^{2 \ell}}=\sum_{n \geq \ell}\left[\left(\begin{array}{c}
n+\ell-1 \\
2 \ell-1
\end{array}\right) \cdot \ell !\right] q^{2 n}
$$

Let us now turn to the first formula of Corollary 7.1. First, we notice that $U_{\mu_{\ell}} \cdots U_{\mu_{1}} \emptyset$ is the linear combination of all ribbon tableaux of content $\mu$, with the sign of the tableau as coefficient. So the left-hand side of the first formula in Corollary 7.1 is given by

$$
\left\langle U_{v_{\ell}} \cdots U_{v_{1}} \emptyset, U_{\mu_{\ell}} \cdots U_{\mu_{1}} \emptyset\right\rangle
$$

which by duality is equal to $\left\langle\emptyset, D_{v_{1}} \cdots D_{\nu_{\ell}} U_{\mu_{\ell}} \cdots U_{\mu_{1}} \emptyset\right\rangle$.

Lemma 9.1 Let $\mu, v$ be two compositions with $\ell$ parts. Then

$$
\left\langle\emptyset, D_{v_{1}} \cdots D_{v_{\ell}} U_{\mu_{\ell}} \cdots U_{\mu_{1}} \emptyset\right\rangle=v_{\ell} \times \sum_{\rho}\left\langle\emptyset, D_{v_{1}} \cdots D_{v_{\ell-1}} U_{\rho} \emptyset\right\rangle,
$$

where $\rho$ runs through the multiset of all compositions of length $\ell-1$ obtained by deleting a part of $\mu$ of size $\nu_{\ell}$.

Proof of the lemma We use the relations of Proposition 9.1, with $D_{v_{\ell}}$ and $U_{\mu_{i}}$, for $i$ equal to $\ell, \ldots, 1$ successively. For a given $i$, two cases can occur: If $\mu_{i} \neq$ $v_{\ell}$, then $D_{v_{\ell}}$ and $U_{\mu_{i}}$ simply commute; otherwise, we have in addition a term $v_{\ell}\left\langle\emptyset, D_{v_{1}} \cdots D_{v_{\ell-1}} U_{\rho} \emptyset\right\rangle$ which has to be added, where $\rho$ is the composition with the part $\mu_{i}$ deleted in $\mu$.

The proof of the first part of Corollary 7.1 is now a simple induction on $\ell$ based on the preceding lemma.

\section{Columns of the character table of the symmetric group}

Ribbon tableaux have a strong connection with the representation theory of the symmetric group, which we will now recall briefly. Let $n$ be a positive integer, $\lambda$ a partition of size $n$; we denote by $\chi^{\lambda}$ the irreducible character of the symmetric group 
$\mathfrak{S}_{n}$ indexed by $\lambda$ (for more information on these topics, see, for instance, $[10,20]$ that have a combinatorial approach). Let also $\chi_{\mu}^{\lambda}$ be the value of this character on a permutation of cycle type $\mu$ : this means that the permutation has $m_{i}$ cycles of length $i$ for each $i$ if $\mu=\left(1^{m_{1}} 2^{m_{2}} \ldots\right)$. The Murnaghan-Nakayama rule [16, 17] is given by:

Theorem 10.1 Let $\lambda, \mu$ be two partitions of the same size $n$. Then

$$
\chi_{\mu}^{\lambda}=\left|R T_{\lambda, \mu}\right|_{ \pm}
$$

In fact, the rule says a bit more: one, in fact, has $\chi_{\mu}^{\lambda}=\left|R T_{\lambda, c}\right|_{ \pm}$for any composition $c$ whose corresponding nonincreasing rearrangement $\tilde{c}$ is given by $\mu$.

This rule gives a combinatorial interpretation of $\chi_{\mu}^{\lambda}$, and shows in particular that it is an integer. We will now show that Theorem 7.2 is adapted to study the column sums of the character table.

10.1 A formula for $\sum_{\lambda} \chi_{\mu}^{\lambda}$

Define $C(\mu)=\sum_{\lambda} \chi_{\mu}^{\lambda}$, the sum of all entries of column $\mu$ in the character table of $\mathfrak{S}_{n}$. By the Murnaghan-Nakayama rule, $C(\mu)$ is equal to the signed sum of all ribbon tableaux of content $\mu$. By Theorem 7.2, this last quantity is itself equal to the signed sum of hook involutions of content $\mu$. The preceding result can thus be summed up by $C(\mu)=\left|R T_{\mu}\right|_{ \pm}=|\mathcal{H} \mathcal{I}|_{ \pm}$. Using Corollary 6.1, we finally obtain

$$
C(\mu)=\left|\mathcal{H} \mathcal{I}_{\text {spec }}(\mu)\right|
$$

This shows in particular that $C(\mu)$ is a nonnegative integer; the following theorem gives a formula for the exact value of this integer.

Theorem 10.2 Let $\mu=\left(1^{m_{1}} 2^{m_{2}} \ldots\right)$ be a partition.Then we have the formula $C(\mu)=\prod_{i>0} c_{i, m_{i}}$ where

$$
c_{i, m_{i}}= \begin{cases}0 & \text { if } i \text { is even and } m_{i} \text { is odd } \\
\left(m_{i}-1\right) ! ! \cdot i^{m_{i} / 2} & \text { if } i \text { is even and } m_{i} \text { is even } \\
\sum_{k=0}^{\left.L \frac{m_{i}}{2}\right\rfloor}\left(\begin{array}{c}
m_{i} \\
m_{i}-2 k
\end{array}\right) \cdot(2 k-1) ! ! \cdot i^{k} & \text { if } i \text { is odd } .\end{cases}
$$

We will exhibit two proofs: first a bijective one, and then algebraic, using the tools of Sect. 9 .

First Proof of Theorem 10.2 The computation of $\left|\mathcal{H} \mathcal{I}_{\text {spec }}(\mu)\right|$ for general $\mu$ clearly reduces to the case $\mu=\left[i^{m_{i}}\right]$ where $\mu$ has only one part size.

In this case, an element of $\mathcal{H I}_{\text {spec }}(\mu)$ is an involution on $\llbracket 1, a_{k} \rrbracket$ with a choice of a hook of size $a_{k}$ for each cycle of length 2. Remembering that elements of $\mathcal{H} \mathcal{I}_{\text {spec }}(\mu)$ have no fixed points corresponding to even parts, we obtain easily the above expression for the coefficient $c_{i, m_{i}}$, and the proof is complete. 
Second proof of Theorem 10.2 We now give a proof that does not use Theorem 7.2 (and thus also not equality (7)). For this, we need the following algebraic consequence of Proposition 6.1 in terms of the operators $D_{i}$ and $U_{i}$ (considered as endomorphisms of $\mathbb{K}^{\mathbf{Y}}$ ); we denote by $\mathbf{Y}$ the vector $\sum_{\lambda \in \mathbf{Y}} \lambda \in \mathbb{K}^{\mathbf{Y}}$, and $o_{i}$ is 1 when $i$ is odd and 0 otherwise.

Proposition 10.1 For all $i \geq 1$, we have $D_{i} \mathbf{Y}=U_{i} \mathbf{Y}+o_{i} \cdot \mathbf{Y}$.

Proof Take the scalar product of each member of the equality with a partition $\lambda$, and remembering that $U_{i}$ and $D_{i}$ are dual operators, the result is equivalent to

$$
\sum_{\mu \in \mathcal{U}_{i}(\lambda)} \varepsilon(\mu / \lambda)=\sum_{\mu \in \mathcal{D}_{i}(\lambda)} \varepsilon(\lambda / \mu)+o_{i} .
$$

This is an immediate corollary to Proposition 6.1.

By the Murnaghan-Nakayama rule, we have $C(\mu)=\left\langle D_{\mu} \mathbf{Y}, \widehat{\emptyset}\right\rangle$. We will use the relations of Propositions 9.1 and 10.1 to compute this scalar product.

Lemma 10.1 We have the following formulas:

1. For $m \geq 2$ and $i \geq 1, D_{i}^{m} \mathbf{Y}=o_{i} \cdot D_{i}^{m-1} \mathbf{Y}+(m-1) i \cdot D_{i}^{m-2} \mathbf{Y}+U_{i} D_{i}^{m-1} \mathbf{Y}$.

2. For $m \geq 1$ and $i \geq 1, D_{i}^{m} \mathbf{Y}=c_{i, m} \mathbf{Y}+U_{i} A_{i, m} \mathbf{Y}$, where $c_{i, m}$ is defined in Theorem 10.2 , and $A_{i, m}$ is a polynomial in $D_{i}$ and $U_{i}$.

3. For $m \geq 1$ and $i \geq 1,\left\langle D_{\mu} D_{i}^{m} \mathbf{Y}, \emptyset\right\rangle=c_{i, m}\left\langle D_{\mu} \mathbf{Y}, \emptyset\right\rangle$ if all parts of $\mu$ are greater than $i$.

Proof of the lemma We have $D_{i}^{m} \mathbf{Y}=o_{i} \cdot D_{i}^{m-1} \mathbf{Y}+D_{i}^{m-1} U_{i} \mathbf{Y}$, thanks to Proposition 10.1. Using $m-1$ times the relation $D_{i} U_{i}=U_{i} D_{i}+i \cdot I$, part 1 of the lemma is proved.

By an immediate induction on part 1, we can write for all $m \geq 2$ that $D_{i}^{m} \mathbf{Y}=$ $b_{i, m} \mathbf{Y}+U_{i} B \mathbf{Y}$ for a certain endomorphism $B$ and an integer $b_{i, m}$ necessarily equal to $\left\langle D_{i}^{m} \mathbf{Y}, \varnothing\right\rangle$. Substituting into part 1, and taking the coefficient of $\emptyset$ in each member, we obtain $b_{i, m}=o_{i} b_{i, m-1}+(m-1) i b_{i, m-2}$. The numbers $c_{i, m}$ verify the same recurrence relation, as can be easily seen, directly or by the combinatorial interpretation given in the first proof. Since we have in addition $b_{i, 0}=c_{i, 0}=1$ and $b_{i, 1}=c_{i, 1}=o_{i}$, it follows that $b_{i, m}=c_{i, m}$ for all $i, m$ and part 2 is proved.

Finally, thanks to part 2, the left-hand side of the equality of part 3 is equal to

$$
c_{i, m}\left\langle D_{\mu} \mathbf{Y}, \emptyset\right\rangle+\left\langle D_{\mu} U_{i} A_{i, m} \mathbf{Y}, \emptyset\right\rangle .
$$

Since $D_{\mu}$ commutes with $U_{i}$ by (4), the second term is then 0 because the image of $U_{i}$ has null intersection with $\mathbb{K} \emptyset$, and the lemma is proved.

The proof of Theorem 10.2 is now immediate by induction on the number of different part sizes of $\mu$, using part 3 in the previous lemma. 


\subsection{Other evaluations of $C(\mu)$}

The formula of Theorem 10.2 is not new, but the proof above is (to the best of our knowledge) the first fully bijective proof of it based on the Murnaghan-Nakayama rule. Let us mention two other places in the literature where this result is shown, and show the equivalence to our formulation.

The computation of $C(\mu)$ is an exercise in Macdonald's book [15, p. 122, Ex. 11], and relies on symmetric function techniques. It is proved that $C(\mu)$ is equal to the product $\prod_{i>1} a_{i}^{\left(m_{i}\right)}$, where $a_{i}^{(m)}$ is the coefficient of $t^{m} /(m !)$ in $\exp \left(t+\frac{1}{2} i t^{2}\right)$ (resp., $\left.\exp \left(\frac{1}{2} i t^{2}\right)\right)$ if $i$ is odd (resp., even). Through an expansion of the series, one checks easily that $a_{i}^{(m)}$ is indeed equal to the coefficient $c_{i, m}$ of Theorem 10.2.

Another proof can be found in Exercise 7.69 of Stanley's book [27]; the proof is based on a general result in character theory, whose specialization to the symmetric group is the following theorem:

Theorem 10.3 $[13,27]$ Let $\sigma$ be a permutation of $\llbracket 1, n \rrbracket$ with cycle type $\mu$. Then $C(\mu)$ is equal to the number of square roots of $\sigma$ in $\mathfrak{S}_{n}$, i.e., to the number of permutations $\tau \in \mathfrak{S}_{n}$ such that $\tau^{2}=\sigma$.

We will give a new proof of this theorem. First, let us fix some notation: given a permutation $\sigma$ of cycle-type $\mu=\left(1^{m_{1}} 2^{m_{2}} \cdots\right)$, consider the decomposition of $\sigma$ into disjoint cycles

$$
\sigma=\left[c_{1}^{(1)} \cdots c_{1}^{\left(m_{1}\right)}\right]\left[c_{2}^{(1)} \cdots c_{2}^{\left(m_{2}\right)}\right] \cdots
$$

where $c_{i}^{(k)}, k=1, \ldots, m_{k}$, are the cycles of length $i$ written in increasing order of their minimal elements. For instance, the permutation 574389216 has cycle type $\mu=$ $\left(3,2^{3}\right)$ and will be written [(27)(34)(69)][(158)].

Proof of Theorem 10.3 Thanks to formula (7), it suffices to exhibit a bijection $\Gamma$ between $\mathcal{H} \mathcal{I}_{\text {spec }}(\mu)$ and $\left\{\tau \mid \tau^{2}=\sigma\right\}$. The following lemma is the key to this bijection.

Lemma 10.2 Let $c_{1}, c_{2}$ be two disjoint cycles of length $m \geq 2$ in $\mathfrak{S}_{n}$.

- There exist exactly $m$ cycles $c$ of length $2 m$ in $\mathfrak{S}_{n}$ such that $c^{2}=c_{1} c_{2}$.

- If $m$ is odd, there exist a unique cycle c of length $m$ in $\mathfrak{S}_{n}$ such that $c^{2}=c_{1}$.

The $m$ cycles of the first part of the lemma will be denoted $\operatorname{root}_{k}\left(c_{1}, c_{2}\right), k=$ $0, \ldots, m-1$, and the unique cycle of length $m$ determined by the second part is $\operatorname{root}\left(c_{1}\right)$. We extend these notations to the case $m=1$ where $c_{1}, c_{2}$ are fixed points $a, b$ by setting $\operatorname{root}_{0}(a, b)$ to be the cycle $(a b)$ and root $(a)$ to be the length 1 cycle $(a)$.

We now consider an element $I \in \mathcal{H} \mathcal{I}_{\text {spec }}(\mu)$; it is readily seen that $I$ can be considered as a sequence of hook involutions $\left(I_{j}\right)_{j \geq 1}$ where $I_{j}$ belongs to $\mathcal{H} \mathcal{I}_{\text {spec }}\left(j^{m_{j}}\right)$. We wish to define a permutation $\tau=\Gamma(I)$ such that $\tau^{2}=\sigma$.

Consider a hook involution $I_{j}$ as above and denote by $i_{j}$ the underlying involution. If $(x, y)$ is a transposition in $i_{j}$, and $h \in \llbracket 0, j-1 \rrbracket$ is the height of the corresponding 
hook, then by the preceding lemma we can define a cycle $C=\operatorname{root}_{h}\left(c_{j}^{(x)}, c_{j}^{(y)}\right)$. If $z$ is a fixed point of $i_{j}$, then $j$ must be odd by definition of $\mathcal{H} \mathcal{I}_{\text {spec }}\left(j^{m_{j}}\right)$, and thanks to the lemma we can in this case define a cycle $C^{\prime}=\operatorname{root}\left(c_{j}^{(z)}\right)$. Now all these cycles $C, C^{\prime}$, over all indices $j$, are pairwise disjoint; their product is the desired $\operatorname{root} \Gamma(I)$ of $\sigma$.

We can also use the formula to answer the question: For a given integer $k$, what are the partitions $\mu$ such that the column sum $C(\mu)$ is equal to $k$ ? Let $\mathcal{O D}$ be the set of partitions with odd distinct parts. The answers for the first integers are:

- $C(\mu)=0$ if and only if $\mu$ has at least an even part with odd multiplicity;

$-C(\mu)=1$ if and only if $\mu \in \mathcal{O D}$;

- $C(\mu)=2$ if and only if 1 has multiplicity 2 and $\mu-1^{2} \in \mathcal{O D}$, or 2 has multiplicity 2 and $\mu-2^{2} \in \mathcal{O D}$;

- $C(\mu)=3$ has no solution;

$-C(\mu)=4$ if and only if 3 has multiplicity 2 and $\mu-3^{2} \in \mathcal{O D}$, or 4 has multiplicity 1 and $\mu-4^{1} \in \mathcal{O D}$, or 2 and 1 have multiplicity 2 and $\mu-1^{2} 2^{2} \in \mathcal{O D}$.

The number of solutions to $C(\mu)=0$ is sequence A085642 in Sloane's Online Encyclopedia [24]. The article [1] proves that another family of partitions is in bijection with $\mathcal{O D}$, namely the partitions with at least one part congruent to 2 modulo 4 .

\section{Extensions}

In this last section, we give three different extensions where the ideas of this work can be applied.

\subsection{A result of Stanton and White}

Stanton and White [28] show combinatorially that, if $c$ and $c^{\prime}$ are 2 compositions verifying $\widetilde{c}=\widetilde{c^{\prime}}$, then

$$
\left|R T_{\lambda, c}\right|_{ \pm}=\left|R T_{\lambda, c^{\prime}}\right|_{ \pm}
$$

This is a consequence of the Murnaghan-Nakayama rule, see Theorem 10.1 and the remark following it: both sides of (8) express the value of the character $\chi^{\lambda}$ on a permutation $\sigma$ of cycle type $\widetilde{c}=\widetilde{c^{\prime}}$. In this section, we give local rules that give a simple proof of Stanton and White's result, building on Fomin's version of jeu de taquin explained in his appendix to Stanley's book [27].

Proof of (8) We have the following proposition whose proof can be easily done by using the encoding of partitions by infinite sequences of elements of $\{0,1\}$ as explained in Appendix:

Proposition 11.1 Let $\lambda, \mu, \xi$ be three partitions such that $\mu / \lambda$ and $\xi / \mu$ are ribbons. Then we separate two cases: 
1. $\xi / \lambda$ is not a ribbon: in this case there exists a unique $\nu \neq \mu$ such that $\nu / \lambda$ and $\xi / \nu$ are ribbons of respective sizes $|\nu / \lambda|=|\xi / \mu|$ and $|\xi / \nu|=|\mu / \lambda|$.

2. $\xi / \lambda$ is a ribbon:

(a) If there exists $v$ such that $v / \lambda$ and $\xi / v$ are ribbons of respective sizes $|\nu / \lambda|=$ $|\xi / \mu|$ and $|\xi / \nu|=|\mu / \lambda|$, then such $a v$ is uniquely defined;

(b) Otherwise, there exists a unique $\widehat{\mu} \neq \mu$ such that $\widehat{\mu} / \lambda$ and $\xi / \widehat{\mu}$ are ribbons of respective sizes $|\widehat{\mu} / \lambda|=|\mu / \lambda|$ and $|\xi / \widehat{\mu}|=|\xi / \mu|$.

Furthermore, we have the sign relations $\varepsilon(\nu / \lambda) \varepsilon(\xi / \nu)=\varepsilon(\mu / \lambda) \varepsilon(\xi / \mu)$ and $\varepsilon(\widehat{\mu} / \lambda) \varepsilon(\xi / \widehat{\mu})=-\varepsilon(\mu / \lambda) \varepsilon(\xi / \mu)$.

This is a generalization of Proposition A1.2.1 in [27], which is the case where $\mu / \lambda$ and $\xi / \mu$ have size 1 .

We can now define the local rules: given $\lambda, \mu, \xi$ as in the proposition, we draw them on a lozenge as on the left of Fig. 13. If a corresponding " $v$ " can be defined according to Proposition 11.1, then we erase $\mu$, and write $v$ on the right vertex of the lozenge; otherwise we replace $\mu$ by $\widehat{\mu}$. This last rule corresponds to a "change of direction" in the global correspondence that we define below, as did rules $\mathrm{S}$ and $\mathrm{T}$ in the Algorithm $\phi$ from Sect. 6 . We also define inverse local rules by a simple vertical symmetry, and the trivial rule which consists of simply moving the partition $\mu$ from one side of the lozenge to the other.

We gather now these lozenges in a grid $P_{\ell}$ made of rows of $1,2, \ldots, \ell-1$ lozenges from top to bottom. We index the lozenges with the coordinates $(i, j), 1 \leq i \leq j \leq$ $\ell-1$, such that the lozenges in the $m$ th row from the top receive the indices (1, $\ell-m),(2, \ell-m+1), \ldots,(m, \ell-1)$ from left to right; see Fig. 13, right, for the case $\ell=4$. We also fix a linear order on lozenges such that each lozenge $(i, j)$ has to be bigger than the two lozenges on its top left and bottom left, namely $(i-1, j)$ and $(i, j-1)$ when they are defined. For the examples, we will use the following linear order: $(i, j)$ is greater than $\left(i^{\prime}, j^{\prime}\right)$ if $i>i^{\prime}$, or $i=i^{\prime}$ and $j>j^{\prime}$.

Given two compositions $c, c^{\prime}$ of length $\ell$ such that $\tilde{c}=\widetilde{c^{\prime}}$, we represent elements of $R T_{\lambda, c}$ on the left side of $P_{\ell}$ as chains of partitions labeling the vertices from bottom to top, and elements of $R T_{\lambda, c^{\prime}}$ on the right side in the same fashion. Now we want to select a subset of the lozenges so that when local rules are applied in the grid we indeed obtain a correspondence between $R T_{\lambda, c}$ and $R T_{\lambda, c^{\prime}}$. Fix $\sigma$, a permutation such that $\sigma(c)=c^{\prime}$, and mark lozenges of $P_{\ell}$ in the following way: for each $j$ such $1<j \leq l$, mark the lozenges $(i, j)$ such that $i \leq\left|\left\{k<j: \sigma_{k}>\sigma_{j}\right\}\right|$.

Fig. 13 Lozenge for local rules and associated grid for the global correspondence
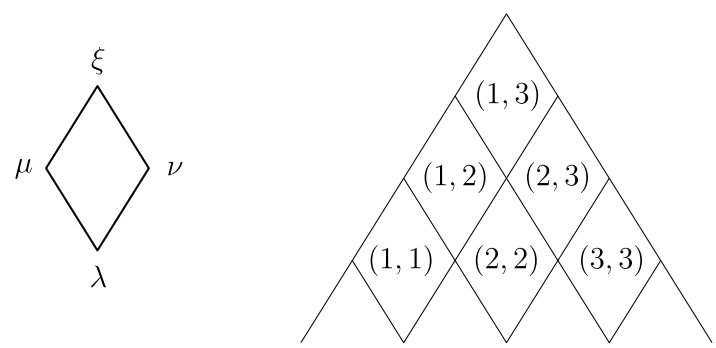

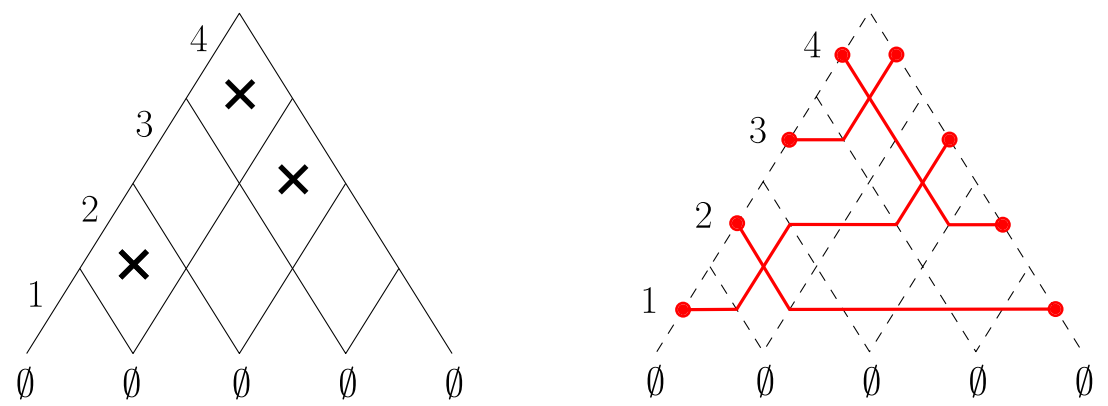

Fig. 14 Marking of the lozenges for the permutation 3142, and resulting effect on the size of ribbons through the correspondence

Fig. 15 Example of the correspondence in the proof of (8)

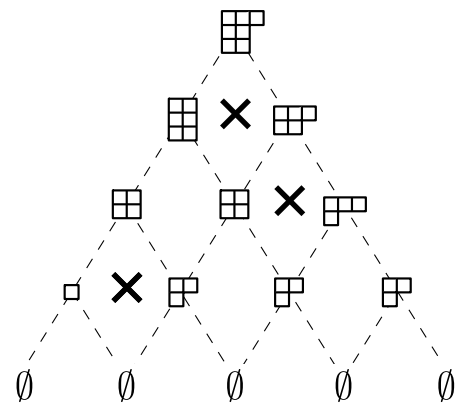

For instance, consider $c=(1,3,2,1)$ and $c^{\prime}=(3,1,1,2)$, and fix the permutation $w=3142$ which indeed verifies $w(c)=c^{\prime}$; the corresponding marking is represented on the left-hand side of Fig. 14.

Now we are in position to give a global correspondence based on the local rules defined above: start from a ribbon tableau represented on the left-hand side, and go on performing local rules as in the beginning of Sect. 6, following the fixed linear order. Here we perform nontrivial local rules in the marked lozenges, while in the other (unmarked) ones we will use only the trivial local rule. An example is given on Fig. 15. The definition of the marked lozenges in the previous paragraph is made so that the size of ribbons "match" between $c$ and $c^{\prime}$, thanks to Proposition 11.1; this can be visualized on the right-hand side of Fig. 14.

This global correspondence is then a signed bijection which proves (8).

Remark 11.1 In the case when all lozenges are marked with a cross, and all ribbons are of size 1, we obtain precisely Schützenberger's involution as described in Fomin's appendix to [27].

\subsection{Other correspondences based on the graph $G R$}

In the correspondence of Theorem 7.1, we considered pairs of ribbon tableaux of the same shape. As already noticed, these are very special paths in the ribbon graph $G R$ : they start and end at $\varnothing$, going up $\ell$ steps and then down $\ell$ steps. The same ideas work 
when building correspondences for other kinds of paths, as is the case in the work of Fomin [7]. Here we show this in the case of oscillating tableaux which are well known in the standard case.

We consider the paths in $G R$ of length $2 \ell$, that start and end at $\varnothing$, and which possess $\ell$ steps up and $\ell$ steps down (but in no imposed order). These are called oscillating tableaux (of shape $\emptyset$ ) in the case where all ribbons are of size 1 , and we will thus call these paths oscillating ribbon tableaux.

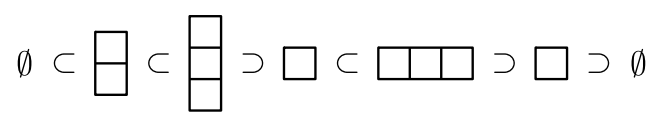

The size $n$ of such a path is the half sum of the sizes of the $2 \ell$ edges (i.e., ribbons), and the sign is the product of the signs of those ribbons. The oscillating tableau above has size 5 , sign +1 , and length 6 . Let us denote by $O s c_{n, \ell}$ the signed set of such paths of size $n$ and length $2 \ell$; we have then the following formula:

$$
\left|O s c_{n, \ell}\right|_{ \pm}=(2 \ell-1) ! !\left(\begin{array}{c}
n+\ell-1 \\
\ell-1
\end{array}\right) .
$$

This can be proved in two ways, algebraic and bijective, following the steps of what was done in the case of pairs of ribbon tableaux of the same shape.

The algebraic way to prove the identity is to notice that the quantity $\left|O s c_{n, \ell}\right|_{ \pm}$can be expressed as the coefficient of $q^{2 n}$ in the series $\left\langle\emptyset,(\mathbf{D}+\mathbf{U})^{2 \ell} \emptyset\right\rangle$. Now this series is $(2 \ell-1) ! !\left(\sum_{i} i q^{2 i}\right)^{\ell}$ (this a consequence of Corollary 2.6 (a) of [25]), and (9) follows as in the proof of the second equality of Corollary 7.1.

The bijective way consists in constructing a signed bijection between $O s c_{n, \ell}$ and hook matchings of $\llbracket 1,2 \ell \rrbracket$ with size $n$ : these are perfect matchings on $\llbracket 1,2 \ell \rrbracket$ such that to each pair $\{i, j\}$ of the matching is associated to a hook $H_{\{i, j\}}$ such that the sum of the sizes of the $\ell$ hooks is $n$. Since there are $(2 \ell-1)$ !! perfect matchings, Proposition 4.1 shows that there are indeed $(2 \ell-1) ! !\left(\begin{array}{c}n+\ell-1 \\ \ell-1\end{array}\right)$ such hook perfect matchings.

The bijective correspondence between $O s c_{n, \ell}$ and hook matchings is done as in Roby [18]. We illustrate this on an example in Fig. 16. Instead of the grid $G_{\ell}$, we perform the bijection on a grid $T_{\ell}$ illustrated on the figure by dashed lines for $\ell=3$.

Hook matchings can be represented by labeling with $\varnothing$ the bottom and left side, and for each pair $\{i, j\}$ of the matching, the corresponding hook is drawn in the square of column $i$ from the left and row $j$ from the top. In the example, the matching is then $\{\{1,3\},\{2,6\},\{4,5\}\}$. Oscillating ribbon tableaux $\left(\lambda_{0}=\emptyset, \lambda_{1}, \ldots, \lambda_{2 \ell-1}, \lambda_{2 \ell}=\emptyset\right.$ are represented on the outside corners of the north east border, from top left to bottom right (moreover, in each of the corresponding inside corners, one draws the smallest shape between $\lambda_{i}$ and $\left.\lambda_{i+1}\right)$.

Now a signed correspondence goes along the exact same lines as what we did in Sect. 6 for pairs of ribbon tableaux of the same shape: We fix a total order on the squares of $T_{\ell}$, and apply local rules in these squares, changing directions when we encounter a rule $\mathrm{S}$ or $\mathrm{T}$; this gives us in the end a bijective proof of (9). The example of Fig. 16 does not present any occurrence of rules $\mathrm{S}, \mathrm{T}$ for the sake of simplicity. 
Fig. 16 Correspondence for oscillating ribbon tableaux

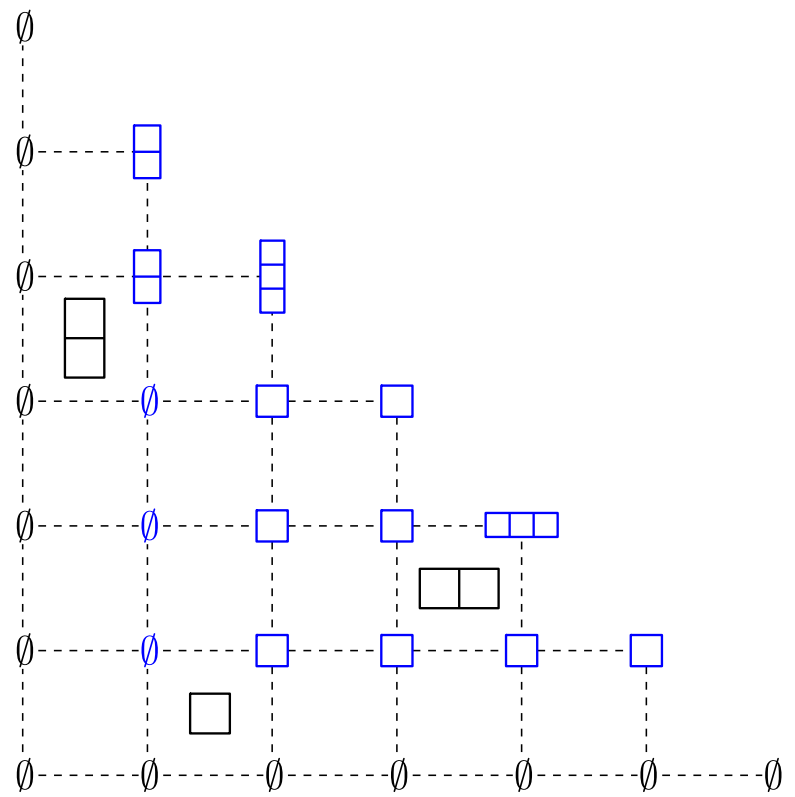

\subsection{Layered graphs in duality}

The techniques used in this article generalize the kind of graphs studied in Fomin's framework developed in $[6,7]$; we now present the theoretical setting in this subsection.

Consider a graph $G=(V, E)$ with a sign function on the edges $\varepsilon: E \rightarrow\{+1,-1\}$. Suppose that $V$ is the disjoint union of finite sets $V_{i}, i \in \mathbb{N}$ where $V_{0}$ is a singleton $\{O\}$. We will say that $G$ is a layered graph (with zero). Let now $U_{i}, D_{j}$ be the endomorphisms of $\mathbb{K} V$ defined for $v \in V_{k}$ by $U_{i}(v)=\sum_{e} \varepsilon(e) v^{\prime}$ where $e$ runs through all edges from $v$ to $v^{\prime} \in V_{k+i}$, and $D_{j}$ is defined dually from $\mathbb{K} V_{k}$ to $\mathbb{K} V_{k-i}$.

We say that the layered graph $G$ is self dual if there exist nonnegative integers $\alpha_{i}, i \geq 1$ such that

$$
\begin{aligned}
& D_{i} U_{i}=U_{i} D_{i}+\alpha_{i} \cdot \mathrm{Id}, \\
& D_{i} U_{j}=U_{j} D_{i} \quad \text { if } i \neq j .
\end{aligned}
$$

Fomin's framework of self dual graded graphs is the case where

- Edges exist only between consecutive levels $V_{i}$ and $V_{i+1}$;

- The sign function is constant equal to 1 .

It is then possible to use the algebraic techniques of this work to study the enumeration of paths in such graphs. The relations above correspond to certain equalities of signed cardinals, as in the graph $G R$. If signed bijections proving these equalities are fixed, then we can determine global correspondences in the same way. But it obviously remains to see if there exists interesting examples to which this theory can be applied. 
An important remark is that this generalizes only Theorem 7.1 and its Corollary; to obtain the counterpart of Theorem 7.2, one needs additional local properties on the self dual layered graph, analogous to Proposition 10.1 in the case of GR.

Acknowledgements The authors would like to thank the anonymous referee for a very thorough reading of the manuscript; a great number of improvements to the original version of the paper are due to him/her.

P.N. is supported partly by the Austrian Science Foundation (FWF), in the framework of the Wittgensteinpreis (Grant Z130-N13) and the National Research Network "Analytic Combinatorics and Probabilistic Number Theory" (Grant S9607-N13).

\section{Appendix: Partitions as sequences of zeros and ones}

In this Appendix, we will show how the encoding of partitions by words is well suited to the study of the operations on ribbons of Sect. 2.2. We follow van Leeuwen [30] for notations. Let $\lambda$ be a partition, and $\delta(\lambda) \in\{0,1\}^{\mathbb{Z}}$ the sequence defined by the following procedure: we extend the top and left borders of a Ferrers diagram to infinity, and read the lower right boundary from bottom to top, recording 1 for every vertical edge encountered, and 0 for the horizontal edges.

For instance, the partition $(4,2,2,1)$ has for coding word $(\cdots 1110101$ | $1001000 \cdots)$, cf. Fig. 17; the sign "|" separates the parts of the border below and above the diagonal of the diagram, and we consider that nonnegative indices of $\delta(\lambda)$ are those on the right of $\mid$. Notice that the encoding sequences have the following characteristic properties (see [30]):

1. They differ from $(\cdots 1111 \mid 0000 \cdots)$ (corresponding to the empty partition) in a finite number of positions;

2. The number of 0 s to the left of $\mid$ is equal to the number of 1 s to its right.

Now, ribbons addable to $\lambda$ (resp., removable from $\lambda$ ) are in bijection with pairs of indices $(i, j)$ in $\mathbb{Z}$ where $i<j$ such that $\delta_{i}(\lambda)=1$ and $\delta_{j}(\lambda)=0$ (resp., $\delta_{i}(\lambda)=0$ and $\left.\delta_{j}(\lambda)=1\right): i$ indicates the position of the head, and $j$ the position of the tail. If $\mu$ is the partition obtained after addition or removal of the ribbon, then $\delta(\mu)$ is the result of the exchange of 0 and 1 at positions $i$ and $j$ in $\delta(\lambda)$.

Fig. 17 Infinite word encoding a partition

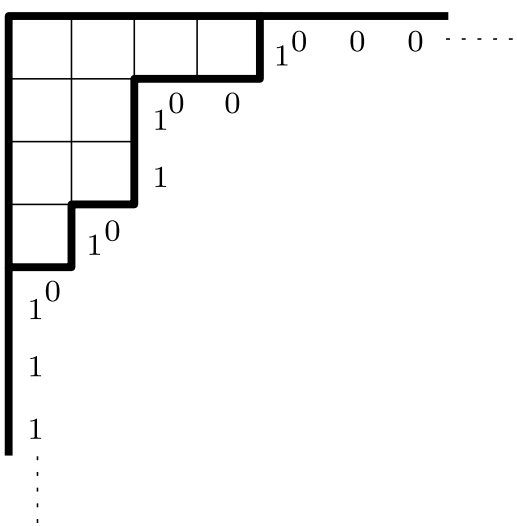




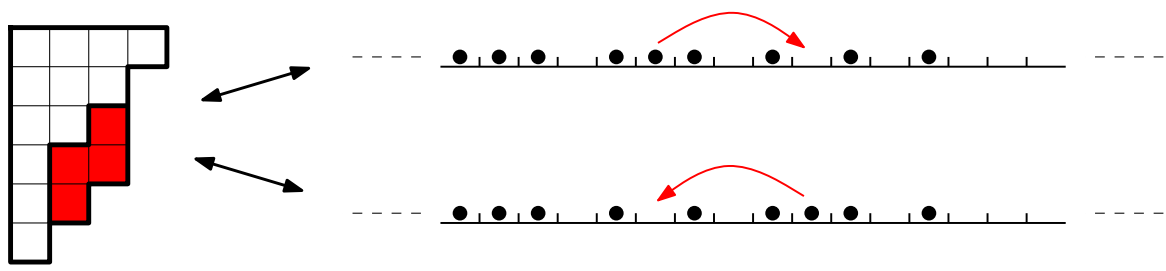

Fig. 18 Addition of a ribbon on $\delta(\lambda)$

One may think of $\delta(\lambda)$ as a configuration of particles on the infinite discrete line: the $1 \mathrm{~s}$ represent particles, and 0 s represent empty positions. So moving a particle in an empty position to its left (resp., right) corresponds to removing (resp., adding) a ribbon. Figure 18 shows a ribbon $\lambda / \mu$ by an arrow between its tail and head, and the codes of $\lambda$ and $\mu$ are given on the right. One has then the following result:

Lemma A.1 Let $\lambda$ be a partition with associated sequence $\delta(\lambda)$, and $i<j$ be the indices of $\delta(\lambda)$ corresponding to a ribbon $r$ addable to (or removable from) $\lambda$, that is, we have $\left\{\delta_{i}(\lambda), \delta_{j}(\lambda)\right\}=\{0,1\}$. Then

1. The size of $r$ is $|r|=j-i$.

2. The height $h$ of $r$ is the number of 1 in $\delta(\lambda)$ between the indices $i$ and $j$, i.e., $h=\mid\left\{k \in \mathbb{Z} \mid i<k<j\right.$ and $\left.\delta_{k}(\lambda)=1\right\} \mid$.

Proof It goes simply by using the fact that the 1 s correspond to vertical steps on the boundary of $\lambda$, and the 0 s to horizontal ones; so $j-i$ is equal to the number of cells occupied by $r$, and each 1 between $i$ and $j$ corresponds to going up from one row to another in the ribbon.

Now the data of $\lambda, \mu, v$ (when $\mu, v \neq \lambda$ ) in a direct rule is equivalent to the data of $\delta(\lambda)$ and integers $i_{1}<j_{1}, i_{2}<j_{2}$ (corresponding to $\mu / \lambda$ and $\nu / \lambda$ ), where $\delta_{i_{1}}(\lambda)=1, \delta_{j_{1}}(\lambda)=0$ and $\delta_{i_{2}}(\lambda)=1, \delta_{j_{2}}(\lambda)=0$.

Every operation of Sect. 2.2 can be, in fact, best understood given this representation; we shall do it for the switchout operation, which corresponds to the local rule $S$ in Sect. 5. Using the notations above, the rule $S$ applies precisely when one of the following two cases occur:

1. $i_{1}=i_{2}, j_{1} \neq j_{2}$ and $\delta_{j_{1}+j_{2}-i_{1}}(\lambda)=1$, or

2. $j_{1}=j_{2}, i_{1} \neq i_{2}$ and $\delta_{i_{1}+i_{2}-j_{1}}(\lambda)=0$.

Let $i$ be the common value of $i_{1}$ and $i_{2}$ in the first case, and $j$ the common value of $j_{1}$ and $j_{2}$ in the second case. Then applying rule $S$ consists simply in defining $\widehat{\lambda}$ as the partition whose code is obtained from $\delta(\lambda)$ by exchanging $0 s$ and $1 s$ at positions $i, j_{1}, j_{2}$ and $j_{1}+j_{2}-i$ in the first case, and at positions $i_{1}, i_{2}, j$ and $i_{1}+i_{2}-j$ in the second case. The first case is illustrated in Fig. 19: $\lambda$ is shown above with the ribbons, and the applications of rule $S$ gives the partition below. (The symbols ' $x$ ' represent indifferently 1 s or 0 s.)

One notices that the partition $\mu / \widehat{\lambda}$ and $\mu / \widehat{\lambda}$ are ribbons of the same size $j_{1}-i_{1}$, and $\nu / \lambda$ and $\nu / \widehat{\lambda}$ are ribbons of the same size $j_{2}-i_{2}$; therefore, $\lambda$ and $\widehat{\lambda}$ belong to 


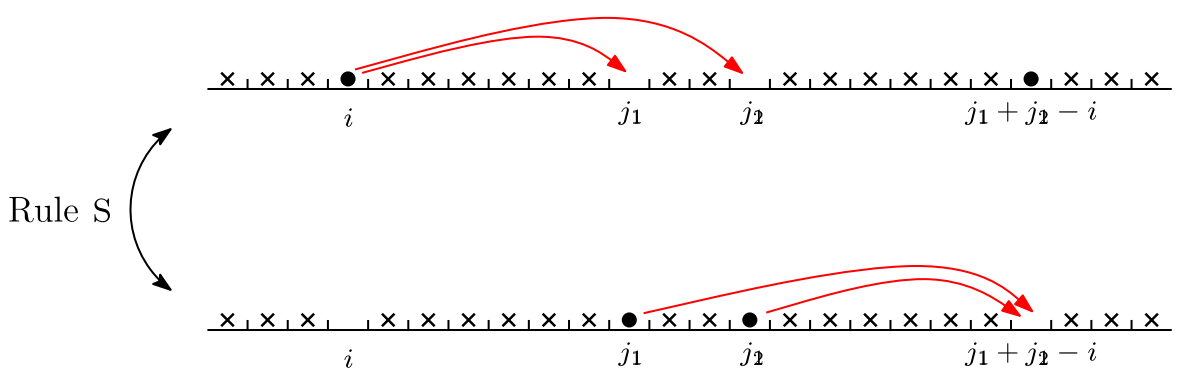

Fig. 19 The rule $S$

the same set $\mathcal{D}_{k}(\mu, v)$ for a certain $k$. The rule $\mathrm{S}$ is also clearly an involution, as it exchanges cases 1 and 2 defined above.

Now we will check finally that it exchanges signs, which is just a matter of counting particles, thanks to Lemma A.1. Since $S$ is involutive, we can assume to be in case 1 above, and, by the symmetry of the roles of $\mu$ and $\nu$, we also assume $j_{1}<j_{2}$ without any loss of generality; we are therefore in the case of Fig. 19, top. Let $a$ (resp., $b, c$ ) be the number of $1 \mathrm{~s}$ in $\lambda$ that are strictly between the indices $i$ and $j_{1}$ (resp., $j_{1}$ and $j_{2}$; resp., $j_{2}$ and $j_{1}+j_{2}-i$ ); these numbers are the same in $\lambda$ and $\widehat{\lambda}$. As elements of the signed set $\mathcal{D}_{j_{1}-i}(\mu, v), \lambda$ and $\widehat{\lambda}$ have signs $(-1)^{x}$ and $(-1)^{y}$, with $x=2 a+b$ and $y=b+2 c+1$; this gives opposite signs since $x$ and $y$ have opposite parity.

\section{References}

1. Bessenrodt, C., Olsson, J.: On the sequence A085642. In: The On-line Encyclopedia of Integer Sequences (2004)

2. Chauve, C., Dulucq, S.: A geometric version of the Robinson-Schensted correspondence for skew oscillating tableaux. Discrete Math. 246(1-3), 67-81 (2002)

3. Dulucq, S., Sagan, B.E.: The Robinson-Schensted correspondence for skew-oscillating tableaux. In: Proceedings of the 4th Conference on Formal Power Series and Algebraic Combinatorics, Amsterdam, The Netherlands, pp. 129-142. Amsterdam, Elsevier (1995)

4. Dulucq, S., Sagan, B.E.: La correspondance de Robinson-Schensted pour les tableaux oscillants gauches. Discrete Math. 139(1-3), 129-142 (1995)

5. Fomin, S.V.: The generalized Robinson-Schensted-Knuth correspondence. Zap. Nauč. Semin. LOMI 155(Differentsialnaya Geometriya, Gruppy Li i Mekh. VIII):156-175, 195 (1986)

6. Fomin, S.: Duality of graded graphs. J. Algebr. Comb. 3(4), 357-404 (1994)

7. Fomin, S.: Schensted algorithms for dual graded graphs. J. Algebr. Comb. 4(1), 5-45 (1995)

8. Fomin, S.: Schur operators and Knuth correspondences. J. Comb. Theory, Ser. A 72(2), 277-292 (1995)

9. Fomin, S., Stanton, D.: Rim Hook Lattices. Algebra Anal. 9(5), 140-150 (1997)

10. Fulton, W.: Young Tableaux. London Mathematical Society Student Texts, vol. 35. Cambridge University Press, Cambridge (1997). With applications to representation theory and geometry

11. Garsia, A., Milne, S.: Method for constructing bijections for classical partition identities. Proc. Natl. Acad. Sci. USA 78(4, part 1), 2026-2028 (1981)

12. Garsia, A., Milne, S.: A Rogers-Ramanujan bijection. J. Comb. Theory, Ser. A 31(3), 289-339 (1981)

13. Isaacs, I.M.: Character Theory of Finite Groups. Dover, New York (1994). Corrected reprint of the 1976 original (Academic Press, New York; MR0460423 (57 \#417))

14. Kerber, A.: Applied Finite Group Actions. Algorithms and Combinatorics, vol. 19, 2nd edn. Springer, Berlin (1999) 
15. Macdonald, I.G.: Symmetric Functions and Hall Polynomials. Oxford Mathematical Monographs, 2nd edn. Clarendon/Oxford University Press, New York (1995)

16. Murnaghan, F.D.: On the representations of the symmetric group. Am. J. Math. 59(3), 437-488 (1937)

17. Nakayama, T.: On some modular properties of irreducible representations of a symmetric group. I. Jpn. J. Math. 18, 89-108 (1941)

18. Roby, T.: The connection between the Robinson-Schensted correspondence for skew oscillating tableaux and graded graphs. Discrete Math. 139(1-3), 481-485 (1995). Formal power series and algebraic combinatorics (Montreal, PQ, 1992)

19. Sagan, B.E.: Shifted tableaux, Schur Q-functions, and a conjecture of R. Stanley. J. Comb. Theory, Ser. A 45(1), 62-103 (1987)

20. Sagan, B.E.: The Symmetric Group. Graduate Texts in Mathematics, vol. 203, 2nd edn. Springer, New York (2001). Representations, combinatorial algorithms, and symmetric functions

21. Sagan, B.E., Stanley, R.P.: Robinson-Schensted algorithms for Skew tableaux. J. Comb. Theory, Ser. A 55(2), 161-193 (1990)

22. Schensted, C.: Longest increasing and decreasing subsequences. Can. J. Math. 13, 179-191 (1961)

23. Shimozono, M., White, D.E.: Color-to-spin ribbon Schensted algorithms. Discrete Math. 246(1-3), 295-316 (2002). Formal power series and algebraic combinatorics (Barcelona, 1999)

24. Sloane, N.: On-line encyclopedia of integer sequences. Accessible from N. Sloane's homepage

25. Stanley, R.P.: Differential posets. J. Am. Math. Soc. 1(4), 919-961 (1988)

26. Stanley, R.P.: Variations on differential posets. In: Invariant Theory and Tableaux, Minneapolis, MN, 1988. IMA Vol. Math. Appl., vol. 19, pp. 145-165. Springer, New York (1990)

27. Stanley, R.P.: Enumerative Combinatorics, vol. 2. Cambridge Studies in Advanced Mathematics, vol. 62. Cambridge University Press, Cambridge (1999). With a foreword by Gian-Carlo Rota and appendix 1 by Sergey Fomin

28. Stanton, D.W., White, D.E.: A Schensted algorithm for rim hook tableaux. J. Comb. Theory, Ser. A 40(2), 211-247 (1985)

29. Sundaram, S.: The Cauchy identity for $\mathrm{Sp}(2 n)$. J. Comb. Theory, Ser. A 53(2), 209-238 (1990)

30. van Leeuwen, M.A.A.: Edge sequences, ribbon tableaux, and an action of affine permutations. Eur. J. Comb. 20(2), 179-195 (1999)

31. White, D.E.: A bijection proving orthogonality of the characters of $S_{n}$. Adv. Math. 50(2), 160-186 (1983) 